B. R. Coriton, A. M. Steinberg, J. H. Frank, "High-Speed Tomographic PIV and OH PLIF Measurements in Turbulent Reactive Flows" Experiments in Fluids 55:1743-62 (2014)

The original publication is available through Springer at:

http://dx.doi.org/10.1007/s00348-014-1743-3 


\title{
High-Speed Tomographic PIV and OH PLIF Measurements in Turbulent Reactive Flows
}

\author{
Bruno Coriton ${ }^{1}$, Adam M. Steinberg ${ }^{2}$, Jonathan H. Frank ${ }^{1^{*}}$ \\ ${ }^{1}$ Combustion Research Facility, Sandia National Laboratories, Livermore, California 94551 \\ ${ }^{2}$ Institute for Aerospace Studies, University of Toronto, Toronto, Ontario, M3H 5 T6
}

Full-length article,

Submitted to Experiments in Fluids on September 10, 2013.

\author{
${ }^{*}$ Corresponding Author \\ Jonathan H. Frank \\ P.O. Box 969, MS 9053 \\ Livermore, CA 94551, USA \\ Fax: (925) 294-2595 \\ jhfrank@sandia.gov
}




\begin{abstract}
High-speed tomographic particle image velocimetry (TPIV) is demonstrated in turbulent reactive flows at acquisition rates ranging from $10 \mathrm{kHz}$ to $16 \mathrm{kHz}$. The $10 \mathrm{kHz}$ TPIV measurements are combined with planar laser-induced fluorescence (PLIF) imaging of $\mathrm{OH}$ to mark the high-temperature reaction zone of the flame. Simultaneous TPIV/OH PLIF measurements are applied to the stabilization region of a weakly-turbulent lifted DME/air jet flame $\left(R e_{D}=7,600\right)$ and the mixing layer of a turbulent partially-premixed DME/air jet flame $\left(R e_{D}=29,000\right)$. In the lifted jet flame, vortical structures exhibit time-dependent morphological changes and eventually dissipate as they approach the flame. In the near field of the turbulent jet flame, dynamics of localized extinction are captured as coherent structures with high compressive strain rates interact with the reaction zone and subsequently break apart. The principal axis of compressive strain has a strong preferential orientation at 45 degrees with respect to the jet axis. The $3 \mathrm{D}$ velocity field measurements are used to evaluate biases in $2 \mathrm{D}$ measurements of compressive strain rates in a turbulent jet flame. The biases in the $2 \mathrm{D}$ measurements primarily stem from out-of-plane orientation of the principal axis of compressive strain. Comparisons with a constant density turbulent non-reactive jet $\left(R e_{D}=22,600\right)$ show that the jet flame has larger coherent structures that are confined near the reaction zone. Data from the non-reactive jet are also used to evaluate effects of noise, bias, and spatial averaging on measurements of the velocity and velocity gradients.
\end{abstract}

Keywords: Tomographic PIV, High speed imaging, OH LIF, Flame, Strain rate. 


\section{Introduction}

Improved fundamental understanding of the structure and dynamics of turbulent flows and turbulence-flame interactions is essential for developing physics-based combustion models and advanced combustion technologies. The inherent three-dimensional nature of turbulent flows requires volumetric measurements to fully capture the structure of these interactions. Moreover, studies of turbulence and turbulent flame dynamics require threedimensional measurements at repetition rates that are fast enough to resolve the relevant flow time scales. Highrepetition rate 3D imaging of the dynamics of turbulent flows and flow-flame interactions poses a significant diagnostics challenge.

Particle image velocimetry (PIV) is commonly used to measure velocity fields in reactive and non-reactive flows. The particular PIV implementation that is used often depends on the optical accessibility of the flow system, along with the availability of cameras and the requisite pulsed laser system. In the conventional planar imaging mode, PIV provides a two-component velocity measurement in the plane of a thin laser sheet using a single camera (Adrian 1991). Stereoscopic particle image velocimetry (SPIV) extends the PIV capability to threecomponent velocity measurements in a plane by using two cameras that view the flow field from different angles (Prasad and Adrian 1993). Both PIV and SPIV techniques have been used extensively in laminar and turbulent combustion studies, where the velocity measurements are typically combined with planar laser-induced fluorescence (PLIF) imaging of a fuel tracer or combustion intermediate species, such as $\mathrm{CH}$ or OH (e.g., Frank et al. 1999, Filatyev et al. 2005; Steinberg et al., 2010; Coriton et al. 2013). The PLIF images of combustion radicals provide a marker of the high-temperature reaction zone whose interplay with the flow field can then be analyzed. The development of high frame-rate CMOS cameras and high repetition-rate lasers has enabled such measurements to be conducted at high speed in turbulent flames, which is necessary for understanding the dynamics of turbulence-flame interactions (Sick 2013). High repetition-rate planar SPIV has been used for pseudo-3D velocity measurements in a low Reynolds number jet flame by invoking Taylor's hypothesis (Ganapathisubramani et al. 2008; Steinberg et al. 2009; Gamba et al. 2013).

The most challenging and equipment intensive PIV implementation is tomographic particle image velocimetry (TPIV), which provides full three-component volumetric velocity measurements using multiple cameras to simultaneously record laser scattering from seed particles at different viewing angles. Previous TPIV applications have primarily focused on non-reactive liquid and gas-phase flows (Elsinga et al. 2008; Scarano 2013). Single-shot TPIV measurements have been demonstrated in a motored internal combustion engine, which did not involve combustion (Baum et al. 2013). Recently, Weinkauff et al., 2013 demonstrated the feasibility of single-shot TPIV measurements in a turbulent lifted jet flame. TPIV measurements offer access to the complete velocity gradient tensor, from which key fluid dynamic quantities, such as the divergence, vorticity, principal strain rate components and tensor invariants, can be determined. In contrast, two-dimensional PIV techniques provide incomplete or biased measurements of these quantities in the presence of three-dimensional flow structures. Volumetric three-component velocity data are critical for proper interpretation and analysis of 
temporally resolved measurements in turbulent flows, wherein three-dimensional motion can greatly influence the interpretation of temporal data sequences (Steinberg et al. 2011). The application of TPIV to turbulent flame studies presents additional challenges of significant variations in particle seeding density due to the wide range of gas densities, increased imaging noise, beam steering from variations in the index of refraction, and the requirements for high framing rates.

In the present study, we demonstrate the feasibility of high-speed volumetric three-component velocity measurements in a lifted jet flame and a turbulent partially-premixed jet flame using TPIV, conducted simultaneously with high-speed OH PLIF measurements. TPIV measurements in a non-reactive jet are first used to evaluate errors and bias in velocity gradients. Second, we investigate the dynamics of flame-vortex interactions at the base of the lifted flame and then compare the distributions of compressive strain rates in the turbulent non-reactive and reactive jets. We also evaluate the ambiguity in the interpretation of strain rate statistics from two-dimensional PIV measurements. Each case study illustrates new capabilities for studies of turbulent flame dynamics as enabled by the three-dimensional, high-speed diagnostics.

\section{Experimental methods}

\subsection{Experimental conditions}

Figure 1 provides a schematic of the experimental configuration for simultaneous high repetition rate TPIV and OH PLIF measurements. The setup consisted of two high repetition rate lasers and five high-speed CMOS cameras that were positioned at different viewing angles. The UV laser beam $(\lambda=283.3 \mathrm{~nm})$ for OH LIF excitation and the visible laser beam $(\lambda=532 \mathrm{~nm})$ for the TPIV measurements were overlapped and aligned with the jet burner axis. Figure 2 shows photographs of the jet burner and flame luminosity from the dimethyl-ether (DME)/air jet flames that were used for demonstrating high-speed imaging measurements. The piloted jet burner was similar to the burner of the Sandia $\mathrm{CH}_{4}$ /air turbulent flame series ( $c f$. TNF workshop) and consisted of a jet nozzle, a pilot annulus and a large coflow with diameters of $D=7.45 \mathrm{~mm}, 18.2 \mathrm{~mm}$ and $203 \mathrm{~mm}$, respectively. The axes of the reference frame are oriented as depicted in Fig. 1, with the $x$-axis parallel to the propagation direction of the laser beams, the $y$-axis parallel to the burner centerline, and the $z$-axis orthogonal to the OH PLIF laser sheet. We denote $v_{x}, v_{y}$ and $v_{z}$ the velocity components of the velocity vector $v$ such that $v=v_{x} \hat{\imath}+v_{y} \hat{\jmath}+$ $v_{z} \hat{k}$, where $(\hat{\imath}, \hat{\jmath}, \hat{k})$ is the unit vector basis of the reference frame.

Measurements were applied to two jet flames: a weakly-turbulent lifted jet flame (Case 1 - Fig. 2a) and a turbulent partially-premixed jet flame (Case 2 - Fig. 2b). In addition, a non-reactive air jet (Case 2NR) with the same volumetric flow rate as Case 2 was studied, in order to compare reactive and non-reactive conditions. A summary of the flow conditions is provided in Table 1 , including the jet mixture equivalence ratio, $\varphi_{j e t}$, the jet exit Reynolds numbers $R e_{D}=V_{j e t} D / v$, the jet and coflow bulk velocities, $V_{j e t}$ and $V_{\text {coflow }}$, respectively, and the 
composition of the pilot gases, if any. The DME/air jet mixtures for Cases 1 and 2 were delivered at jet Reynolds numbers, $R e_{D}$, of approximately 7,600 and 29,000, respectively. For the non-reactive turbulent jet (Case 2NR), the jet exit velocity was matched to that of the reactive jet (Case 2), which resulted in a lower jet Reynolds number of 22,600 because of the higher kinematic viscosity of air compared to DME. The air coflow velocity for all cases was $0.9 \mathrm{~m} / \mathrm{s}$. For the lifted jet flame (Case 1), an air stream was flowing through the pilot annulus, which caused the flame to remain detached from the nozzle. For the attached jet flame (Case 2), the flame was stabilized by an annulus of lean premixed pilot flames consisting of a mixture of $\mathrm{C}_{2} \mathrm{H}_{2}, \mathrm{H}_{2}$, air, $\mathrm{CO}_{2}$, and $\mathrm{N}_{2}$ with the same enthalpy and post-flame equilibrium composition of a 0.60 equivalence ratio DME/air flame; no pilot flow was used for Case $2 N R$.

The locations of measurements in the various jets are indicated by rectangular boxes in Figs. 2a and 2b. For Case 1, $10 \mathrm{kHz}$ TPIV and OH PLIF measurements were performed in the stabilization region of the lifted flame at a distance of $y / D=11.5$ above the jet nozzle exit. For Case 2, simultaneous TPIV/OH PLIF measurements at $10 \mathrm{kHz}$ were recorded at a distance of $y / D=20$ above the nozzle in a region that overlapped the turbulent reaction zone of the partially-premixed jet flame. Additionally, $16 \mathrm{kHz}$ TPIV measurements, without OH PLIF, were collected for Cases 2 and $2 N R$ at $y / D=10$. These measurements were used for a comparison of coherent structures in reactive and non-reactive turbulent jets presented in Section 4.2.

Table 1 - Summary of flow conditions for the DME/air jet flames

\begin{tabular}{c|ccccc}
\hline Case & $\boldsymbol{\varphi}_{\text {jet }}$ & $\boldsymbol{R e}_{\boldsymbol{D}}$ & $\begin{array}{c}\boldsymbol{V}_{\text {bulk }} \\
(\mathbf{m} / \mathbf{s})\end{array}$ & $\begin{array}{c}\boldsymbol{V}_{\text {coflow }} \\
(\boldsymbol{m} / \mathbf{s})\end{array}$ & Pilot \\
\hline 1 (Fig. 2a) & 12.4 & 7,600 & 8.4 & $\sim 0.9$ & Air \\
2 (Fig. 2b) & 3.6 & 29,000 & 45.9 & $\sim 0.9$ & Lean flame \\
2NR & 0 (Air) & 22,600 & 45.9 & $\sim 0.9$ & None \\
\hline
\end{tabular}

\subsection{Tomographic Particle Image Velocimetry (TPIV) system}

The experimental configuration for tomographic PIV consisted of a high repetition rate laser and four highspeed CMOS cameras (Fig. 1). The jet and air coflow were seeded with $0.3 \mu \mathrm{m}$ aluminum oxide particles, which was small enough to ensure good tracking of the flow. The particle laden flow field was illuminated using a $\mathrm{Nd}$ :YAG laser with two independently controlled heads, each of which provided $50 \mathrm{~W}$ at a pulse repetition rate of $10 \mathrm{kHz}$ (Quantronix, Dual-Hawk-HP). The overlapping laser beams were collimated and cropped with a rectangular slit aperture to select the most uniform portion of the beam. The resulting laser sheet had less than $20 \%$ variation in energy across the width of the reconstructed probe volume, thereby providing nearly uniform illumination of the particles. The beams from the two laser heads were orthogonally polarized, and the polarization angles were rotated to approximately 45 degrees with respect to the plane of the cameras to provide comparable Mie scattering signal from each laser beam onto the cameras. Laser light scattering from the particles 
in the illuminated volume were simultaneously imaged onto four high-speed CMOS cameras (Vision Research, Phantom V1610), using identical macro camera lenses (Tamron, f.l. $=180 \mathrm{~mm}$ ). As shown in Fig. 1, two pairs of cameras were positioned on each side of the laser volume, and the four cameras were arranged such that each camera was oriented at an angle of approximately 25 degrees relative to the normal to the laser sheet. The camera lenses were mounted at angles relative to the detector plane using Scheimpflug mounts to compensate for the misalignment of the image and object planes for each camera,. Opposing cameras had slightly different viewing angles, as well as different off-axis perspectives. The lens apertures were set to $f / 22$ and $f / 16$ on the lenses that collected light scattered in the forward and backward directions, respectively. These aperture settings provided the necessary depth of field for imaging particles throughout the probe volume. The different aperture settings compensated for the higher efficiency of forward scattering, such that comparable signal levels were recorded on all cameras. The acquisition of the four cameras was synchronized with the Nd:YAG laser triggering such that particle scattering from each laser pulse was recorded on a single frame of each camera in a frame-straddling mode.

The laser heads were triggered at repetition rates of $10 \mathrm{kHz}$ for the simultaneous TPIV and OH PLIF measurements and $16 \mathrm{kHz}$ for the TPIV measurements without the OH PLIF imaging. The time delay between pulses from each laser head was $20 \mu$ s for measurements in the lifted jet flame (Case 1), and $4 \mu$ s and $6 \mu$ s for measurements in the turbulent partially-premixed jet flame (Case 2) at $y / D=10$ and 20, respectively. A $4 \mu$ s time delay was also used for the measurements in the turbulent non-reactive jet (Case $2 N R$ ) at $y / D=10$.

The timing was configured such that each pair of laser pulses for the PIV measurement straddled sequential frames on the cameras. As a result, the cameras operated at framing rates of $20 \mathrm{kHz}$ and $32 \mathrm{kHz}$ for laser repetition rates of $10 \mathrm{kHz}$ and $16 \mathrm{kHz}$, respectively. Camera resolution at $20 \mathrm{kHz}$ was $1024 \mathrm{x} 768 \mathrm{px}^{2}$, which provided a $18.4 \times 11.9 \mathrm{~mm}^{2}$ dewarped field of view in the central plane of the laser volume. At $32 \mathrm{kHz}$, the camera resolution was reduced to $640 \times 688 \mathrm{px}^{2}$, corresponding to a dewarped field of view $11.8 \times 10.7 \mathrm{~mm}^{2}$ in the laser volume midplane.

\subsection{TPIV vector calculation}

The calculation of velocity vectors from the tomographic PIV data was performed using commercial software and consisted of the following steps: camera volume calibration, preprocessing of the particle images, reconstruction of the particle distribution in $3 \mathrm{D}$, and determination of the local velocity vectors followed by vector post-processing. Examples of a reconstructed particle distribution and 3D velocity field are shown in Figs. 3a and $3 \mathrm{~b}$, respectively, for a single-measurement in the stabilization region of the lifted jet flame in Fig. $2 \mathrm{a}$.

For volume mapping and spatial calibration of the four cameras, images of a transparent thin-film target containing an array of dots were recorded on each camera. Target images were recorded with the target located at 13 equally spaced positions along the $z$-axis in order to minimize the error in the calibration throughout the entire volume. The $z$-axis origin position $(z=0.0 \mathrm{~mm})$ corresponded to the position of the OH PLIF laser sheet. The 
calibration was further refined by a volume self-calibration procedure using the measured particle images (Wieneke 2008), resulting in an average mapping function error of approximately 0.06 pixel. To evaluate the effect of variations in the calibration between data sets, we performed separate self-calibrations for the nonreactive (Case $2 N R$ ) and reactive (Case 2) turbulent jets at $y / D=10$. The velocity vectors in the reactive jet were then re-calculated using the self-calibrations from the reactive and non-reactive cases, $\boldsymbol{v}_{(\text {calib } 2)}$ and $\boldsymbol{v}_{(\text {calib } 2 N R)}$, respectively. The relative differences in each velocity component, $\left|1-v_{i,(\text { calib } 2)} / v_{i,(\text { calib } 2 N R)}\right|$, averaged over 500 frames and the entire probe volume were $2.9 \%$ for $v_{x}, 0.6 \%$ for $v_{y}$ and $4.8 \%$ for $v_{z}$. Variations in calibration between the non-reactive flow and flame could result from beam-steering effects due to the index-of-refraction gradients in the flame. The extent of the beam steering effects depends on multiple factors including the size, geometry, and turbulence level of the flame, the path lengths of the laser beam and the light scattered by the particles, as well as the collection optics of the imaging system. Weinkauff et al. (2013) reported a negligible remaining self-calibration disparity of less than 0.2 pixels in their lifted jet flame experiment. A previous study of highly resolved Rayleigh scattering imaging measurements in a turbulent jet flame similar to that of Case 2 used a ray tracing analysis to estimate the deviation of light rays traversing the jet flame (Kaiser et al. 2005). In that study, the estimated spread in the distribution of rays at the detector was on the order of \pm 0.1 pixels at half the maximum of the distribution, although the collection optics and pixel size differed from those of the current study.

Preprocessing of the raw particle images prior to the particle volume reconstruction significantly improved the quality of the resulting velocity vectors. Non-uniformity of the image background was corrected by subtracting the local signal minima in $15 \times 15 \mathrm{px}^{2}$ sub-regions of the particle images. A $3 \times 3 \mathrm{px}^{2} \mathrm{Gaussian}$ smoothing of the particle images was also used to reduce the yield of spurious velocity vectors. The 3D particle positions within the probe volume were reconstructed using four iterations of a Multiplicative Algebraic Reconstruction Tomography (MART) algorithm (Elsinga et al. 2006). The dimensions of the reconstructed volume for the different data sets are listed in the summary of the TPIV parameters in Table 2. The reconstructed volume for $10 \mathrm{kHz}$ TPIV measurements was 17.0 x $11.5 \times 3.4 \mathrm{~mm}^{3}$, resulting in $1133 \times 768 \times 228$ voxels (vx). For the $16 \mathrm{kHz}$ TPIV measurements, the camera resolution was reduced in order to achieve the higher framing rate. As a result, the size of the probe volume was reduced to $11.0 \times 10.5 \times 3.2 \mathrm{~mm}^{3}$, resulting in $669 \times 702 \times 208$ vx. The reconstructed particle distribution in Fig. 3a displays the particle images on the six surfaces of the probe volume using thresholding transparency of the Mie-scattering signal. For clarity, only a subset of the particles is shown. The variation in particle density across the flame front is evident in the top left corner of the probe volume.

Inter-frame particle displacements were determined by performing multi-pass cross-correlation analysis using a final interrogation region size of $24 \times 24 \times 24 \mathrm{vx}\left(360 \times 360 \times 360 \mu \mathrm{m}^{3}\right)$ with a $75 \%$ overlap of neighboring regions, resulting in a $90 \mu \mathrm{m}$ vector spacing and yielding 919,296 vectors in the probe volume for the $10 \mathrm{kHz}$ measurements and 454,454 vectors for the $16 \mathrm{kHz}$ measurements. The sample vector field in Fig. $3 \mathrm{~b}$ shows only 1 out of 64 vectors for viewing clarity. Spurious velocity vectors were detected using a universal outlier detection method (Westerweel and Scarano 2005) and replaced by the average of the $3 \times 3 \times 3$ neighboring vectors. In 
reactive and non-reactive cases, the average number of detected outliers was $11 \%$ of the total number of vectors. A spatial filter based on a penalized least squares method (Garcia 2010) was employed to diminish the noise prior to computing the velocity derivatives. The effect of spatial filtering will be demonstrated in Section 3.3. In Fig. 3b, the high velocity flow near the jet axis is evident on the right-hand side of the probe volume. The region of low velocity $(\sim 1.0 \mathrm{~m} / \mathrm{s})$ at the bottom left corresponds to the air coflow. The increased velocity in the upper left of the probe volume is the flame product region, which also corresponds to the region of lower particle density in Fig. 3a. Note that, for some of the analysis of velocity gradient uncertainty in Section 3.3, different interrogation box sizes and overlaps were employed.

Table 2 - Summary of TPIV measurement parameters

\begin{tabular}{c|ccc}
\hline \multirow{2}{*}{ Case } & $\begin{array}{c}\text { Rep. rate } \\
(\boldsymbol{k H z})\end{array}$ & $\begin{array}{c}\text { Laser head } \\
\text { pulse delay } \\
(\boldsymbol{\mu s})\end{array}$ & $\begin{array}{c}\text { Probe volume size } \\
\left(\mathbf{m m}^{3}\right)\end{array}$ \\
\hline $\boldsymbol{I}$ & 10 & 20 & $17.0 \times 11.5 \times 3.4$ \\
$2-\boldsymbol{y} / \boldsymbol{D}=\mathbf{1 0}$ & 16 & 4 & $11.0 \times 10.5 \times 3.2$ \\
$2-\boldsymbol{y} / \boldsymbol{D}=\mathbf{2 0}$ & 10 & 6 & $17.0 \times 11.5 \times 3.4$ \\
$2 \boldsymbol{N} \boldsymbol{R}$ & 16 & 4 & $11.0 \times 10.5 \times 3.2$ \\
\hline
\end{tabular}

\subsection{OH PLIF imaging}

Planar laser-induced fluorescence (PLIF) imaging of $\mathrm{OH}$ at a repetition rate of $10 \mathrm{kHz}$ was used for measurements of flame topography. For LIF excitation, the second harmonic of a Nd:YAG-pumped dye laser (Edgewave IS8II Nd:YAG, Sirah Credo Dye ) was tuned to a wavelength of $283.3 \mathrm{~nm}\left(\mathrm{E}_{\text {laser }}=175 \mu \mathrm{J} / \mathrm{pulse}\right)$ to excite the $\mathrm{Q}_{1}(7)$ transition of the $\mathrm{A}-\mathrm{X}(1,0)$ band of $\mathrm{OH}$. This laser beam was expanded in the vertical direction using a cylindrical telescope (not displayed in Fig. 1) and formed into a sheet using a cylindrical lens (f.1. $=500$ $\mathrm{mm}$ ). The focused UV beam was overlapped with the $532 \mathrm{~nm}$ laser beam using a dichroic mirror, with the $283.3 \mathrm{~nm}$ laser sheet positioned in the central plane of the volume illuminated by the $532 \mathrm{~nm}$ laser beam. The triggering of the dye laser was configured such that each dye laser pulse was bracketed by a pair of laser pulses for the TPIV measurement. The OH PLIF signal was recorded using a high speed intensified CMOS camera (Vision Research, Phantom V7.3) with a projected pixel size of $37 \times 37 \mu \mathrm{m}^{2}$. The OH PLIF images were not corrected for beam profile variations but were normalized by the peak LIF signal within each image to account for shot-to-shot fluctuations in the laser energy. Registration of the OH PLIF measurement plane within the TPIV probe volume was performed by recording images of a thin-film transparent target on the TPIV and OH PLIF cameras with the target positioned in the plane of the $283.3 \mathrm{~nm}$ laser sheet. The target image of the OH PLIF camera was matched with a bilinear geometric warping algorithm to the target images recorded with the TPIV cameras that were first dewarped in the mid-plane of the probe volume at $\mathrm{z}=0.0 \mathrm{~mm}$. 


\section{Assessment of velocity measurements}

\subsection{Spatial and Temporal Resolution}

In designing experiments for studying the temporal evolution of flow structures, it is important to evaluate the fidelity with which the measurements capture the dynamics of different fluid dynamic scales. First, we consider the spatial resolution of the TPIV measurements relative to fluid dynamic scales. PIV analysis has inherent spatial averaging since each velocity vector is determined from the displacement of many particles distributed over an interrogation region using a cross-correlation (Scarano 2003; Lavoie et al. 2007). This spatial averaging can introduce a velocity bias in the presence of velocity gradients (Keane and Adrian 1990). In PIV measurements of laboratory-scale turbulent flows, it is often difficult or impossible to resolve the finest turbulence scales in the velocity field, and the effects of spatial averaging must be considered. Based on a series of simulations of isotropic turbulence, Worth et al. (2010) suggested that TPIV measurements with a spatial resolution larger than three times the Kolmogorov scale underestimate the vorticity. Although the spatial resolution of a PIV measurement can be improved by reducing the size of the interrogation region, the corresponding increase in the noise level of the cross-correlations of the particle images within the interrogation regions limits the useable resolution. Accordingly, Foucaut et al. (2004) formulated a criterion for optimizing the window size, and thus the measurement resolution, with respect to the spectral noise. However, such an optimization requires a priori knowledge of the turbulence spectrum.

The smallest resolved spatial scales are approximately twice the interrogation box size, which in the present

study corresponds to $\lambda_{m}=720 \mu \mathrm{m}$. For the flow conditions in Table 1, the Kolmogorov scales are approximately $50 \mu \mathrm{m}$ for Case land $30 \mu \mathrm{m}$ for Case $2 N R$ according to the dissipative lengthscale measurements in turbulent non-reactive jets of Frank and Kaiser (2010). For Case 2, the Kolmogorov scale is expected to range between $70 \mu \mathrm{m}$ and $90 \mu \mathrm{m}$ according to measurements of the Batchelor lengthscale in turbulent piloted methane/air flames with a Reynolds number comparable to that of Case2 (Wang et al. 2007). In flames, the local resolution requirements vary significantly since dissipation length scales increase substantially at elevated temperatures (Kaiser and Frank 2009, Frank and Kaiser 2010). Tsurikov and Clemens (2002) found that lengthscales associated with kinetic energy dissipation in turbulent non-reactive jets are between $1 \eta$ and $10 \eta$. The kinetic energy dissipation scales are therefore expected to be under-resolved in the present TPIV measurements.

The integral scale, defined as the full width at half-maximum of the mean axial velocity profiles, ranges from $9 \mathrm{~mm}$ to $18 \mathrm{~mm}$ for the flow conditions in Table 1. The measurement probe volume spanned only half of the jet width since the field of view was centered on the jet shear layer. As a result, the full width of the velocity profile was determined by measuring the half width of the radial profile of the mean axial velocity and doubling the value since the mean flow field was axisymmetric. The spatial dynamic range of the TPIV system is determined by the 
ratio of the probe volume dimensions to the size of the smallest resolved scales. For a constant imaging magnification, the $\mathrm{x}$ and $\mathrm{y}$ dimensions of the probe volume are limited by the CMOS camera resolution. As a result, the spatial dynamic range decreases at higher measurement frequencies because of the lower CMOS camera resolution at higher frame rates. The complete range of turbulence lengthscales, which increases with the turbulent Reynolds number, cannot be entirely captured with the limited spatial dynamic range of TPIV measurements.

Next, we consider how well matched the spatial and temporal scales of the TPIV measurements are for the flows considered here. Two key considerations for any high-repetition-rate experiment are the temporal resolution of the measurement and the residence time of structures in the field-of-view. The adequacy of the temporal resolution can be determined from the ratio of the inter-measurement time, $\Delta t_{m}$, and the turn-over time of the smallest resolved turbulent structures, $\tau\left(\lambda_{m}\right)$, viz.

$$
C_{e}=\frac{\Delta t_{m}}{\tau\left(\lambda_{m}\right)}
$$

The $C_{e}$ parameter is analogous to the Courant-Friedrichs-Lewy (CFL) number employed in simulations and, according the Nyquist criterion, should be less than $1 / 2$ if the experiment is to resolve the dynamics of the smallest measured spatial scales. According to Kolmogorov's theory of turbulence microscales, the time-scale associated with any scale, $\lambda$, is

$$
\tau(\lambda)=\left(\frac{\lambda^{2}}{\epsilon}\right)^{1 / 3} \sim \frac{\lambda^{2 / 3} \delta^{1 / 3}}{u^{\prime}}
$$

Combining Eqs. 1 and 2 gives,

$$
C_{e}=\Delta t_{m} \frac{u^{\prime}}{\lambda_{m}^{2 / 3} \delta^{1 / 3}}
$$

The inter-measurement time is equal to the period of the PIV measurements, which ranged from $0.1 \mathrm{~ms}(10 \mathrm{kHz}$ measurements) to $0.0625 \mathrm{~ms}$ ( $16 \mathrm{kHz}$ measurements) in the current experiments. The RMS velocity fluctuations for the different flow conditions varied from $1 \mathrm{~m} / \mathrm{s}$ to $8 \mathrm{~m} / \mathrm{s}$. The relative temporal resolutions for the different conditions are $C_{e} \sim 0.05$ for the lifted jet flame (Case 1) and $C_{e} \sim 0.25$ for the partially-premixed jet flame and the air jet (Cases 2 and 2NR). These values of $C_{e}$ indicate that the smallest measureable spatial scales were well resolved in time.

The residence time, $\tau_{\text {res }}=l_{F O V} / \bar{v}_{y}$, of a particular scale in the field-of-view can be quantified by 


$$
D_{e}(\lambda)=\frac{\tau_{r e s}}{\tau(\lambda)}=\frac{u^{\prime}}{\bar{v}_{y}} \frac{l_{F O V}}{\lambda^{2 / 3} \delta^{1 / 3}},
$$

where $l_{F O V}$ and $\bar{v}_{y}$ are the length of the field-of-view and mean velocity in the primary flow direction, respectively. $D_{e}(\lambda) \geq 1$ indicates that a structure of size $\lambda$ turns over at least once within the field-of-view. In the present measurements, $D_{e}\left(\lambda_{m}\right) \sim 0.8-1.0$, indicating that the smallest resolved structures did not quite complete a full turn-over as they traversed the field-of-view in the axial direction. In the present experiment, the primary limitations to increasing the value of $D_{e}\left(\lambda_{m}\right)$ are the dimensions and performance characteristics of the CMOS camera. Note that the cameras used here have the best currently available specifications for frame rate versus resolution.

The ratio of parameters $D_{e}\left(\lambda_{m}\right) / C_{e}\left(\lambda_{m}\right)=\tau_{r e s} / \Delta t_{m}$ indicates how well the dominant flow dynamics are resolved This ratio is equal to the number of measurements that are acquired during the mean flow-through time of the probe volume. For the present measurements, the ratio $D_{e}\left(\lambda_{m}\right) / C_{e}\left(\lambda_{m}\right)$ varies from $\sim 25$ in the lifted jet flame (Case 1) to $\sim 4$ in the turbulent jets (Case 2 and 2NR). A small value of $D_{e}\left(\lambda_{m}\right) / C_{e}\left(\lambda_{m}\right)$ corresponds to measurements that are poorly temporally resolved.

\subsection{Evaluation of velocity gradients in turbulent jets}

Measurements of key quantities of interest in turbulent flows, such as vorticity and strain rate, involve the determination of velocity gradients. In principle, the complete velocity gradient tensor is available from TPIV measurements of the three-dimensional velocity field. In practice, the accurate measurement of velocity gradients presents a challenge because calculations of derivatives are sensitive to measurement noise and errors. In this section, we consider the effects of errors and noise on velocity gradient measurements using TPIV results from the turbulent non-reactive jet (Case 2NR).

Uncertainties in the velocity gradients can arise in each stage of the TPIV measurement and analysis. Some sources of error, such as the spatial averaging mentioned in Section 3.1, are intrinsic to the PIV technique and may create biases in accelerating or decelerating flows (Westerweel 2008). Another source of error is the layout of the velocity vectors on a uniformly-spaced grid. The uniform grid does not account for deviations in the actual vector positions that stem from the use of adaptive interrogation regions for determining particle displacements. For TPIV, additional errors in the velocity result from artifacts in the tomographic reconstruction, such as ghost particles, which are replicas of actual particles that result from ambiguities in the reconstruction. The density of ghost particles increases as the particle seeding density increases. According to Atkinson et al. (2011), the presence of velocity gradients accentuates the velocity error associated with ghost particles, whose apparent velocity is given by the mean displacement of real particles in the probe volume. These errors lead to an underestimation of the velocity gradient magnitude (Elsinga et al., 2011). Studies using simulations of TPIV measurements have reported particle displacement measurement errors of 0.1-to-0.4 pixels, although imaging 
noise was not included in these simulations (Elsinga et al. 2006; Worth et al. 2010; Atkinson et al. 2011). Velocity gradient errors are amplified by noise in the particle images and the resulting cross-correlations that are used for determining particle displacements. Filtering techniques are used to reduce the effects of noise in PIV measurements prior to computing velocity derivatives. In flames, additional sources of error in velocity gradient measurements must be considered. For example, thermophoretic effects can introduce a velocity measurement bias in the presence of steep temperature gradients. Typically, thermophoretic biases for PIV measurements in atmospheric pressure flames are on the order of a few $\mathrm{cm} / \mathrm{s}$ (Gomez and Rosner 1993). Beam steering effects also introduce small biases in the measured velocity, as previously mentioned in Section 2.3.

The turbulent air jet is well-suited for evaluating errors in velocity gradient measurements since the mass conservation condition in a constant density flow requires that the flow divergence is zero, $\nabla \cdot \boldsymbol{v}=0$. The divergence can be determined from the three-dimensional velocity field measurements provided by TPIV. If the measured divergence in a constant density flow deviates from zero, it is an indication of errors in the measured velocity gradients. We use the squared divergence to quantify the error in the velocity gradients and compare it to the enstrophy. Figure 4 a shows isosurfaces of squared divergence at $(\nabla \cdot v)^{2}=500 \times 10^{6} \mathrm{~s}^{-2}$ for four instances during a $188 \mu$ s sequence of measurements in the air jet (Case 2NR) that was recorded at $16 \mathrm{kHz}$. These structures of apparent divergence are not well correlated from frame to frame. This lack of persistence is corroborating evidence that the structures in the divergence field are indeed random errors in the divergence measurement and are not associated with actual flow structures. Figure $4 \mathrm{~b}$ shows isosurfaces of both the squared divergence and the enstrophy fields $\left(\omega^{2}=(\nabla \cdot v)^{2}=500 \times 10^{6} \mathrm{~s}^{-2}\right)$ for the same time sequence. In contrast to the divergence field, the coherent structures of enstrophy persist in time as they are convected downstream by the jet flow.

A joint probability density function (PDF) of $\omega^{2}$ and $(\nabla \cdot v)^{2}$ for the TPIV measurements in the turbulent nonreactive jet (Case 2NR) at $y / D=10$ is plotted in Fig. 5. The joint PDF indicates that regions of large apparent divergence preferentially correlate with small values of enstrophy. In contrast, regions of large enstrophy tend to have small values of apparent divergence. These correlations indicate that steep velocity gradients tend to be measured more accurately than small gradients. Previous studies have reported similar results of apparent divergence using TPIV (Scarano and Poelma 2009; Worth et al. 2010; Baum et al. 2013) and other threedimensional velocity measurement techniques (Zhang et al. 1997; Mullin and Dahm 2006; Ganapathisubramani et al. 2007).

\subsection{Effects of Spatial Averaging}

The effects of spatial averaging on the measured enstrophy and apparent divergence are assessed by varying the dimensions of the PIV interrogation region and the smoothing kernel that is used to reduce noise in the velocity measurements. Figure 6 shows the effect of different interrogation region sizes on PDFs of apparent divergence from a set of 500 measurements in Case $2 N R$ at a downstream location of $y / D=10$ and radial positions spanning $x / D=0.1$ to 1.4. The velocity derivatives were computed using a second-order central 
difference scheme within the interior of the measurement volume and first-order differencing schemes along the measurement volume surfaces, following Hearst et al. (2012) and Foucaut and Stanislas (2002). For each interrogation box size, the overlap of the interrogation regions was varied in order to maintain the distance between neighboring vectors at either $180 \mu \mathrm{m}$ (Fig. 6a) or $360 \mu \mathrm{m}$ (Fig. 6b). The resulting PDFs of apparent divergence are symmetric about zero and show a systematic narrowing as the interrogation region dimensions increase from $24 \times 24 \times 24 \mathrm{vx}\left(360 \times 360 \times 360 \mu \mathrm{m}^{3}\right)$ to $48 \times 48 \times 48 \mathrm{vx}\left(720 \times 720 \times 720 \mu \mathrm{m}^{3}\right)$. This progression of decreasing apparent divergence with increasing interrogation region results from a combination of noise reduction and spatial averaging effects. However, a comparison of Figs. 6a and $6 \mathrm{~b}$ shows that there is an even greater reduction in the apparent divergence from an increased vector separation in the central differencing scheme. For an increase in vector spacing from $180 \mu \mathrm{m}$ to $360 \mu \mathrm{m}$, the RMS deviation of apparent divergence decreases from $5.8 \times 10^{3} \mathrm{~s}^{-1}$ to $3.1 \times 10^{3} \mathrm{~s}^{-1}$ with a $24 \times 24 \times 24 \mathrm{vx}$ interrogation volume and from $4.1 \times 10^{3} \mathrm{~s}^{-1}$ to $2.6 \times 10^{3} \mathrm{~s}^{-1}$ for a $48 \mathrm{x} 48 \mathrm{x} 48 \mathrm{vx}$ interrogation volume. For these TPIV measurements, the width of the PDF of apparent divergence is more sensitive to the vector spacing than to the interrogation box size.

Spatial smoothing of the velocity field can be employed to reduce the noise in the velocity gradient measurements. We evaluate the effects of smoothing on the measured enstrophy and squared divergence using TPIV measurements in the shear layer of the non-reactive turbulent jet (Case $2 N R$ ) at $y / D=10$. Velocity derivatives were computed after applying the smoothing filter to the velocity vector field. Smoothing of the velocity vectors was performed using the penalized least squares method of Garcia (2010) that was recently demonstrated to be effective for PIV data (Garcia 2011). The degree of smoothing is governed by a single smoothing strength parameter, $s$. Garcia (2011) showed that smoothing strengths of $s=0.1$ and 0.5 have the same $-3 \mathrm{~dB}$ cutoff frequencies as $2 \times 2 \times 2$ and $3 \times 3 \times 3$ kernels of moving average filters, respectively. This smoothing procedure is also robust to clusters of outlying vectors. We illustrate the effects of smoothing strength by filtering a single-shot velocity field measurement with $s$ ranging from 0.1 to 2.0. The variation in the measured enstrophy and the squared divergence with progressively increasing levels of smoothing are shown by isosurfaces at $\omega^{2}=(\nabla \cdot v)^{2}=1,000 \times 10^{6} \mathrm{~s}^{-2}$ in Fig. 7a and isosurfaces at $\omega^{2}=(\nabla \cdot v)^{2}=500 \times 10^{6} \mathrm{~s}^{-2}$ in Fig. 7b. The images in Fig. 7 show regions of elevated apparent divergence clustered around the structures of elevated enstrophy. As the isosurface level is reduced from $1,000 \times 10^{6} \mathrm{~s}^{-2}$ to $500 \times 10^{6} \mathrm{~s}^{-2}$, additional structures are revealed in the enstrophy and squared divergence fields. The regions of elevated apparent divergence tend to vanish as the smoothing strength is increased. The smallest structures of large enstrophy are also reduced with increased smoothing. However, some coherent structures remain nearly unchanged by the spatial smoothing and persist in all the snapshots in Fig. 7. These structures also remain coherent in time and can be tracked from frame to frame, as previously shown by the time sequence in Fig. 4.

For a quantitative evaluation of the effects of smoothing, we investigate the variations in measured flow statistics using different smoothing strengths. We consider the effects of smoothing on the measured fluctuations of each velocity component over a sequence of TPIV measurements in the turbulent non-reactive jet. The RMS velocity fluctuations were computed for increasing values of the smoothing strength parameter $(s=0.1,05,1.0$ 
and 2.0) and the results are plotted in Fig. 8a. For comparison, the effect of smoothing on fluctuations in the enstrophy and squared divergence are plotted in Fig. 8b. Results for $s=0$ correspond to unsmoothed data. To determine the fluctuations plotted in Figs. $8 \mathrm{a}$ and $8 \mathrm{~b}$, we computed the standard deviation of each quantity at every location in the probe volume across 500 frames of a TPIV measurement time-sequence. The average standard deviation for the time-sequence was then calculated over the entire probe volume. Figure 8a shows that the effect of smoothing on the velocity fluctuations is modest. When $s$ is increased from 0.1 to 2.0 , fluctuations of $v_{x}, v_{y}$ and $v_{z}$ decrease by $3.1 \%, 2.2 \%$ and $6.9 \%$, respectively. The greater sensitivity of the out-of-plane velocity component, $v_{z}$, to smoothing may result from the larger measurement error of the out-of-plane component. In contrast, the fluctuations of enstrophy and squared apparent divergence decrease significantly as the smoothing parameter is increased. The decrease in apparent divergence corresponds to a suppression of noise. Figure $8 \mathrm{~b}$ shows that the fluctuations decrease by $67 \%$ for $\omega^{2}$ and $70 \%$ for $(\nabla \cdot v)^{2}$ when $s$ is increased from 0 to 0.1 , indicating that a small amount of smoothing has a significant effect. Further smoothing results in fluctuation reductions of $48 \%$ for $\omega^{2}$ and $62 \%$ for $(\nabla \cdot v)^{2}$, when $s$ is increased from 0.1 to 2.0. The enstrophy fluctuations are larger than the fluctuations of the squared apparent divergence.

Spatial filtering attenuates the large values of apparent divergence and therefore reduces the error in estimating the local gradients. If the smoothing strength is too high, however, the smallest structures in the turbulent flow will be eliminated. Methods for determining the optimal smoothing strength, such as a spectral analysis of velocity gradients (Foucaut and Stanislas 2002), require analysis that is beyond the scope of this study. For all results presented in Section 4, the velocity gradients are calculated after filtering the velocity vectors using a smoothing strength of $s=2.0$, which has the equivalent $-3 \mathrm{~dB}$ cutoff frequency of a $4 \times 4 \times 4$ moving average filter. The effective reduction in resolution due to smoothing is significantly less than four times the interrogation volume size since the velocity vectors are determined using $75 \%$ overlap of interrogation regions in the TPIV analysis.

\section{Applications to the dynamics of turbulent jet flames}

4.1. Vortex-flame interaction in a lifted jet flame

A significant advantage of high-speed TPIV is that it enables tracking of the time history of flow field structures as they interact with the flame. We demonstrate this capability with simultaneous $10 \mathrm{kHz}$ TPIV and OH PLIF measurements in the stabilization region of a lifted jet flame (Case 1). The frame sequence in Fig. 9 shows the temporal evolution of the velocity and vorticity fields with simultaneous OH PLIF images superimposed to identify the flame product location. The time interval separating each 3D snapshot is $0.5 \mathrm{~ms}$, which corresponds to every fifth frame from the $10 \mathrm{kHz}$ TPIV/OH PLIF measurements (cf. movie in Online Resource 1). For clarity, the velocity vectors are only displayed within the central plane of the measurement 
volume. The vorticity field is represented by enstrophy isosurfaces at $\omega^{2}=15 \times 10^{6} \mathrm{~s}^{-2}$, which correspond to localized regions of high vorticity. Note that all components of the vorticity vector are available from the TPIV, as opposed to only one component from conventional or stereoscopic PIV. The three-dimensional high-speed velocity measurements enable analysis of the shape and orientation of the vortical structures and their evolution in time. Most of the vortical structures are located in the jet shear layer, which can be identified in Fig. 9 by the large radial gradients in the magnitude of the velocity vectors near $x / D=-1$. In the latter portion of the time sequence in Fig. 9, large pockets of high enstrophy emerge from the bottom of the probe volume and are convected upward by the jet flow. These vortical structures remain coherent as they convect downstream, although their morphologies evolve in time. The ability to measure the temporal coherence of vortical structures is indicative of the quality of the TPIV measurements.

A portion of vortical structures directly interact with the flame front. For instance, a vortex tube located in the middle of the probe volume and identified by a red box in the first snapshot in Fig. 9 is convected toward the flame front. At the beginning of the time sequence (top-left image), this vortical structure has a diameter of approximately $2.0 \mathrm{~mm}$ based on the $\omega^{2}=15 \times 10^{6} \mathrm{~s}^{-2}$ isosurfaces. The vortical structure shrinks and loses intensity as it approaches the flame front. Near the end of the time sequence, this vortical structure has almost entirely dissipated. The dissipation of this structure is consistent with the thermal expansion and increased viscosity of the high temperature gases in the flame front region. The vortical structure dissipates before its core penetrates into the region containing flame products, as marked by the OH LIF signal. The thermal expansion creates a region of flow dilation, which results in an acceleration of gases between the reactant and product regions of the flame.

High speed TPIV measurements enable analysis of the interaction between coherent structures and a flame in a frame of reference convecting with the structure. We can thus track the evolution of coherent structures as they are transported by the flow field. This approach is demonstrated for the vortex tube interacting with the lifted flame in Fig. 9. In each frame of the time-sequence, the vortex center was identified as the location of maximum enstrophy in the core of the vortex at the central plane $(z=0.0 \mathrm{~mm})$. The center of the vortex was used as the origin of the reference frame moving with the vortex. We can view the velocity field in this moving frame of reference by subtracting the velocity at the center of the vortex from the velocities that were measured in the laboratory frame of reference. The sequence of snapshots in Fig. 10 shows the evolution of the vortex tube with velocity vectors in the moving frame of reference plotted in the midplane at $\mathrm{z}=0.0 \mathrm{~mm}$. Note that the time origin in Fig. 10 is the same as in Fig. 9. At time $t=-0.7 \mathrm{~ms}$, the vortex tube is composed of two branches that merge into a single vortex tube at $\mathrm{t}=1.0 \mathrm{~ms}$. Subsequently, the size of the vortex tube diminishes as it approaches the base of the flame. The rotational motion in the velocity field surrounding the enstrophy isosurface clearly indicates that the vortical structure extends to a larger region of the flow. To further investigate the vortex evolution as it approaches the flame, we show the temporal evolution of the vortex strength and morphology along with the distance between the vortex core and the flame front in Fig. 11. The maximum enstrophy in the core of the vortex and the vortex-flame distance are plotted as a function of time. For time $t<0 \mathrm{~ms}$, the enstrophy in the core of the vortex increases, possibly as a result of the merging of the two branches of the vortex tube. 
Subsequently, the enstrophy in the core of the single vortex tube decreases as the vortex is convected toward the flame at an average relative velocity of $0.7 \mathrm{~m} / \mathrm{s}$. This decrease in enstrophy likely results from viscous dissipation that is enhanced in the vicinity of the flame.

In planar PIV measurements, only the out-of-plane component of the vorticity vectors can be computed. The tracking of such flow field structures would be ambiguous because their disappearance could result from either the dissipation of vorticity or out-of-plane motion if the structure were convected across the laser sheet and out of the measurement region. In TPIV, the thicker laser sheet reduces the probability that a flow structure will be convected completely out of the probe volume and it provides a mechanism for tracking its motion across the measurement volume. In addition, the ability to measure the cross-plane velocity component in TPIV provides a measure of the out-of-plane convection.

\subsection{Dynamics of flow structures in a turbulent partially-premixed jet flame and a non-reactive jet}

We demonstrate the promise of high-speed TPIV measurements for studying the dynamics of turbulent flames and providing comparisons with turbulent non-reactive flows. We consider TPIV measurements in the turbulent DME/air piloted jet flame (Case 2) and the non-reactive air jet (Case 2NR), which have the same nozzle exit velocity. Turbulent reactive and non-reactive jets have been studied extensively using planar imaging measurements (e.g., Rehm and Clemens 1998; Donbar et al. 2001; Frank et al. 2002; Han and Mungal 2003; Kim and Yoon 2007). In the present study, we use high-speed tomographic PIV to investigate the effects of heat release on the 3-D structure and temporal evolution of the shear layer in turbulent jets. We first consider the flow structures in single-shot measurements shown in Fig. 12, where the three-dimensional velocity vectors are displayed in the midplane (at $z=0.0 \mathrm{~mm}$ ) and blue isosurfaces of enstrophy are shown for $\omega^{2}=500 \times 10^{6} \mathrm{~s}^{-2}$. These snapshots are temporally uncorrelated and are representative of the phenomenology of the reactive and non-reactive turbulent jets. At $y / D=10$, velocities near the jet centerline are typically higher in the flame than in the non-reactive jet because of the greater rate of entrainment of the surrounding air for the non-reactive jet. In the non-reactive jet, the magnitude of the centerline velocity varies between $20 \mathrm{~m} / \mathrm{s}$ and $60 \mathrm{~m} / \mathrm{s}$, whereas the centerline velocity in the flame is significantly more uniform. The spatial distribution and shape of the vortical structures are noticeably different between the two cases. In the non-reactive jet, the enstrophy structures are distributed across a wide region of the jet mixing field. In the jet flame, however, large sheets of high enstrophy are concentrated in the shear layer where air and fuel mix. Coherent structures of high enstrophy generally are absent from the center of the jet flame. In the non-reactive jet, vortical structures are noticeably smaller than those in the turbulent flame, and some structures are small enough to be contained entirely within the probe volume. These findings are consistent with previous studies that showed larger length scales in the dissipation field of turbulent jet flames compared with those in non-reactive jets having comparable jet exit Reynolds numbers (Frank and Kaiser 2010). 
Fluid dynamic strain affects the local reaction rate in flames and can induce localized extinction if the strain rate is sufficiently large and the interaction time with the reaction zone is sufficiently long. In turbulent jet flames, regions with large compressive strain rates and large enstrophy tend to coincide with the location of the reaction zone (Rehm and Clemens 1999). We analyze the dynamics of interactions between compressive strain and the reaction zone in the turbulent jet flame (Case 2) from the $10 \mathrm{kHz}$ TPIV measurements at $y / D=20$. To isolate fluid dynamic strain from dilatation in the flame, we consider the deviatoric strain rate tensor, $S_{o}=$ $\frac{1}{2}\left(\nabla v+\nabla v^{\mathrm{T}}\right)-\frac{1}{3}(\nabla \cdot v) I_{3}$, where $\mathrm{I}_{3}$ is the $3 \times 3$ identity tensor. The deviatoric strain-rate tensor can be characterized by the principal strain rates $\alpha_{o}, \beta_{o}$ and $\Gamma_{o}$, which are eigenvalues of $S_{o}$ and ordered such that $\alpha_{o} \geq \beta_{o} \geq \Gamma_{o}$. The eigenvalues $\alpha_{o}$ and $\Gamma_{o}$ are the extensive and compressive principal strain rates, respectively, and their values are limited to $\alpha_{o} \geq 0$ and $\Gamma_{o} \leq 0$ since the trace of the deviatoric strain-rate tensor is zero everywhere, even in the reactive flow.

The sequence of measurements in Fig. 13 shows the temporal evolution of the velocity, compressive principal strain rate, and $\mathrm{OH}$ PLIF fields during a localized extinction event. The velocity and $\mathrm{OH}$ PLIF signal are displayed in the central plane, and the compressive strain rate is represented by blue isosurfaces at $\Gamma_{o}=-15 \times 10^{3} \mathrm{~s}^{-}$ 1. The onset of extinction occurs at time $\mathrm{t}=0.20 \mathrm{~ms}$ and is identified by the formation of a discontinuity in the contour of the OH PLIF signal. The image sequence shows large coherent structures with high compressive strain rates in the region of localized extinction. These structures remain coherent and closely coupled to the reaction zone until extinction occurs. The large compressive strain rate along the reaction zone results from a parcel of flow that impinges on the flame from the jet side and pushes it towards the coflow. As the flame approaches extinction, the high-temperature reaction zone becomes thinner. Once the flame is locally quenched, the strain rate decreases and the large coherent structures break apart. The residence time of the extinguished region within the probe volume is approximately $0.5 \mathrm{~ms}$, which is too short to capture a subsequent reignition. Additional time sequences showing the dynamics of flame interactions with structures of large compressive strain rate are available in Online Resource 2, where several localized extinctions can be seen to correlate with large compressive strain rates.

The snapshot at time $t=0.20 \mathrm{~ms}$ from Fig. 13 is re-plotted in Fig. 14 with black lines indicating the principal axes of compressive strain, which are given by the local eigenvector of the compressive strain rate eigenvalue. In Fig. 14, only the axes for large compressive strain rates $\left(\Gamma_{o} \leq-15 \times 10^{3} \mathrm{~s}^{-1}\right)$ are displayed. Along the flame extinction region, the compressive strain rate axes are approximately orthogonal to the reaction zone within the $x y$ plane, confirming that the reaction zone is compressed by the local flow. In the same region, the principal axis of the extensive strain (not displayed) is oriented approximately parallel to the flame surface and within the $x y$ plane. The process of strain-induced localized extinction can be related to that of a counterflow flame with increasing strain rate. For this comparison, laminar flame calculations were performed using Chemkin OPPDIF (Kee et al. 2007) and the DME chemical mechanism from Zhao et al. (2008). The predicted laminar flame extinction strain rate is an order of magnitude lower than the compressive strain rate of $-15 \times 10^{3} \mathrm{~s}^{-1}$ that is measured at extinction in 
the turbulent flame. This difference is consistent with the observation that the high-temperature reaction zone is robust to high transient strain-rates (Egolfopoulos 1994).

Statistics on the compressive strain rate in the turbulent partially-premixed flame (Case 2) and the turbulent non-reactive jet (Case 2NR) were computed from 500 frames of the TPIV measurements at $y / D=10$ and are compared in Figs. 15a and 15b. Figure 15a shows PDFs of deviatoric and non-deviatoric compressive strain rates, $\Gamma_{\mathrm{o}}$ and $\Gamma$, respectively. We first consider the deviatoric compressive strain rate. The PDFs of $\Gamma_{\mathrm{o}}$ in the reactive and non-reactive jets are very similar with peaks located at approximately $\Gamma_{\mathrm{o}}=-4 \times 10^{3} \mathrm{~s}^{-1}$. The appearance of structures containing high compressive strain rates, such as those in Figs. 13 and 14 for $\Gamma_{\mathrm{o}}$ exceeding $-15 \times 10^{3} \mathrm{~s}^{-1}$, is an intermittent and localized event that contributes to the tails of these PDFs. The angular orientations of the principal axis of $\Gamma_{\mathrm{o}}$ for both reactive and non-reactive jets are plotted in Fig. 15b. The orientation angles, $\Theta_{\Gamma \mathrm{o}}$, are defined as the angles between the $y$-axis and the projection of the principal axes of compressive strain in either the $x y$ plane (solid curves in Fig. 15b) or the $z y$ plane (dashed curves in Fig. 15b). PDFs of $\Theta_{\Gamma \mathrm{o}}$ are conditioned on large values of the compressive strain rate, $\Gamma_{\mathrm{o}} \leq-15 \times 10^{3} \mathrm{~s}^{-1}$. In the flame, the principal axis of compressive strain has a strong preferential orientation at 45 degrees toward the coflow. Previous studies of turbulent jet flames using two-dimensional measurements also showed a preferential orientation of the principal axes of compressive strain at 45 degrees toward the coflow (Rehm and Clemens 1998; Kothnur et al. 2002). The PDF of the projection of the principal axis in the $z y$ plane has a broader distribution with a peak at zero degrees, indicating a preferential orientation that is parallel to the jet axis. Therefore, the principal axis predominantly lies within the $x y$ plane. In the non-reactive jet, the principal axis of compressive strain remains preferentially oriented at 45 degrees toward the coflow, but the distribution of angles is broader than in the flame. The flame thus enhances the strength of the preferential orientation. Similarly, previous studies using planar laser Rayleigh scattering have shown that dissipation structures have a stronger preferential orientation in turbulent jet flames than in non-reactive turbulent jets (Frank and Kaiser 2010).

\subsection{Comparison of $2 \mathrm{D}$ and $3 \mathrm{D}$ measurements of compressive strain rate}

The 3-D measurements of compressive strain rate are used to assess the limitations and biases of calculating strain rates from 2-D PIV measurements. One limitation is the inability to distinguish between deviatoric and non-deviatoric strain since 3-D velocity field measurements are required to determine the flow divergence. To determine the contribution of flow divergence to the strain rates measured with TPIV, we compare the PDFs of deviatoric and non-deviatoric compressive strain rates in Fig. 15a. The non-deviatoric compressive strain rate, $\Gamma$,

corresponds to the smallest eigenvalue of the strain rate tensor, $S=\frac{1}{2}\left(\nabla v+\nabla v^{T}\right)$. In the non-reactive flow, the two strain rates should be identical because of the zero divergence. However, the PDF of $\Gamma$ extends to small positive values as a result of the apparent divergence from measurement errors in the velocity gradients. In the reactive flow, the positive values of $\Gamma$ result from a combination of measurement errors and dilatation in the 
flame. Although $\Gamma_{\mathrm{o}}$ is strictly negative as a result of the subtraction of the isotropic dilatation term $\frac{1}{3}(\nabla \cdot \boldsymbol{v}) I_{3}$ in $S_{o}$, the errors in the compressive strain rate PDF are not entirely eliminated since the error in velocity gradients is not necessarily isotropic (Ganapathisubramani et al. 2008). Note that the subtraction of the dilatation term has no effect on the angular distribution of the principal strain rate axis in Fig. 15b since the dilatation term is an isotropic tensor.

A significant source of bias in 2D measurements of strain is the ambiguity produced variations in the out-ofplane orientation of the principal strain axes. To evaluate these biases, we simulated a two-dimensional velocity measurement by re-computing the strain rate in the turbulent jet flame (Case 2) using only the velocity components along the $x$ and $y$-axes, thereby intentionally omitting the velocity component along the $z$-axis. The $2 \mathrm{D}$ compressive strain rate, $\gamma^{2 \mathrm{D}}$, was defined as the minimum eigenvalue of the $2 \mathrm{D}$ strain rate tensor, viz.

$$
S^{2 D}=\frac{1}{2}\left(\begin{array}{cc}
2 v_{x, x} & v_{x, y}+v_{y, x} \\
v_{x, y}+v_{y, x} & 2 v_{y, y}
\end{array}\right)
$$

Note that $\mathrm{S}^{2 \mathrm{D}}$ is the non-deviatoric strain rate tensor and must be compared to the non-deviatoric 3D strain rate tensor. Figure 16a shows a joint PDF of the 2D and 3D non-deviatoric compressive strain rates from 500 shots of the TPIV measurements in the turbulent jet flame (Case 2) at $y / D=10$. The results show that the apparent compressive strain rate from $2 \mathrm{D}$ measurements can be very different from the $3 \mathrm{D}$ compressive strain rate. The $2 \mathrm{D}$ measurements systematically under-estimate the local compressive strain rate, although the discrepancy between $2 \mathrm{D}$ and $3 \mathrm{D}$ strain rate measurements decreases for large values of compressive strain rate. The bias in the 2D measurements results from the out-of-plane orientation of the principal axis of the compressive strain with respect to the $x y$ plane. This error is most significant when the principal compressive strain rate axis is nearly aligned with the z-axis. In Fig. 15b, the PDF of the orientation angle of the principal compressive strain axis in the $z y$ plane, $\Theta_{\Gamma o}^{z y}$, shows a substantial probability of non-zero angles, which would produce this bias in the strain rate.

The impact of the out-of-plane orientation of the principal axis on $\gamma^{2 \mathrm{D}}$ is further demonstrated using a joint PDF of $\gamma^{2 \mathrm{D}}$ and the angular orientation within the $z y$ plane, $\Theta_{\Gamma}^{z y}$. The joint PDF in Fig. 16b shows that the probability of measuring positive values of $\gamma^{2 \mathrm{D}}$ increases non-linearly as a function of $\left|\Theta_{\Gamma}^{z y}\right|$. The error in measuring the compressive strain rate increases as $\left|\Theta_{\Gamma}^{z y}\right|$ approaches 90 degrees, which corresponds to alignment of the compressive strain rate principal axis with the z-axis. For $\left|\Theta_{\Gamma}^{z y}\right|=90$ degrees, $\gamma^{2 \mathrm{D}}$ no longer corresponds to the compressive strain rate but is a measure of $\beta$, the intermediate eigenvalue of $S$, which can have either positive or negative values. These biases in $2 \mathrm{D}$ measurements are important to evaluate, particularly since 3D measurements are not practical for all flow facilities.

\section{Summary}


The feasibility of high-speed tomographic PIV measurements in reactive flows was demonstrated at $10 \mathrm{kHz}$ and $16 \mathrm{kHz}$. The $10 \mathrm{kHz}$ velocity measurements were combined with synchronous OH PLIF imaging to enable tracking of the reaction zone in time and its interaction with the flow field. The $3 \mathrm{D}$ velocity measurements were used to determine the nine components of the velocity gradient tensor, from which the enstrophy and strain rate were derived. The results indicate that the structures of high enstrophy and strain rate remain spatially-coherent as they are convected by the flow field.

High-speed TPIV has the potential to unveil new insights into the dynamics of turbulent flames at both the fundamental and practical levels. Simultaneous TPIV/OH PLIF measurements were applied to the stabilization region of a weakly-turbulent lifted DME/air jet flame and a turbulent partially-premixed DME/air jet flame. Results showed the morphological evolution and dissipation of a vortex tube as it interacted with the base of the lifted flame. In the turbulent jet flame, the dynamics of high compressive strain rate structures were captured in regions of localized extinction. The orientation of the principal axis of compressive strain was preferentially oriented at 45 degrees with respect to the jet axis, as previously demonstrated using planar imaging.

Results from the turbulent jet flame were compared with measurements in a constant density turbulent air jet having the same jet exit velocity. The heat release in the flame produced a distinctly different 3-D structure of the enstrophy field. In the flame, regions of high enstrophy formed highly connected coherent structures that were confined to regions near the reaction zone. In contrast, the structures of high enstrophy in the air jet were smaller and dispersed over a wider region of the flow field. The distributions of compressive strain rate were similar in the reactive and non-reactive jets. The principal axis of compressive strain in the non-reactive jet was also preferentially oriented at 45 degrees to the jet axis. However, the preferential orientation in the flame was significantly stronger than that of the non-reactive jet.

The TPIV measurements were used to assess the effect of 3D flow structures on 2D measurements of compressive strain. The 2D measurements of compressive strain underestimate the $3 \mathrm{D}$ non-deviatoric compressive strain rate as a result of out-of-plane orientation of the principal axis of compressive strain. In addition, the 2D measurements cannot be corrected for flow dilatation, which can only be accounted for using 3D velocity measurements. The ability to understand biases from 2D measurements of turbulent flows may be particularly valuable since restricted optical access limits the use of tomographic PIV in some flow facilities.

Errors in determining velocity gradients were observed as non-zero apparent divergence in the constant density turbulent air jet. Structures of elevated apparent divergence were temporally uncorrelated, indicating that they resulted from measurement noise. Spatial averaging in the velocity gradient measurements substantially decreased the apparent divergence. The measured velocity fluctuations were less sensitive to spatial filtering than the velocity gradients. Further work is needed to assess the accuracy of velocity derivatives from tomographic PIV measurements. 


\section{Acknowledgments}

The authors gratefully acknowledge the support of the US Department of Energy, Office of Basic Energy Sciences, Division of Chemical Sciences, Geosciences, and Biosciences. Sandia National Laboratories is a multiprogram laboratory operated by Sandia Corporation, a Lockheed Martin Company, for the US Department of Energy under contract DE-AC04-94-AL85000. A. Steinberg acknowledges the support of the US Air Force Office of Scientific Research under Grant No. FA9550-13-1-0070, Project Monitor Dr. Chiping Li. 


\section{References}

Adrian, RJ (1991) Particle-imaging techniques for experimental fluid mechanics. Annu. Rev. Fluid Mech. 23:261-304

Atkinson, C, Coudert, S, Foucaut, JM, Stanislas, M, Soria, J (2011) The accuracy of tomographic particle image velocimetry for measurements of a turbulent boundary layer. Exp. Fluids 50:10311056

Baum, E, Peterson, B, Surmann, C, Michaelis, D, Böhm, B, Dreizler, A (2013) Investigation of the 3D flow field in an IC engine using tomographic PIV. Proc. Combust. Inst. 34:2903-2910

Chong, MS, Perry, AE, Cantwell, BJ (1990) A general classification of three-dimensional flow fields. Phys. Fluids 2:765-777

Coriton, B, Frank, JH, Gomez A (2013) Effects of strain rate, turbulence, reactant stoichiometry and heat losses on the interaction of turbulent premixed flames with stoichiometric counterflowing combustion products. Combust. Flame, DOI:10.1016/j.combustflame.2013.05.009

Donbar, JM, Driscoll, JF, Carter, CD (2001) Strain rates measured along the wrinkled flame contour within turbulent non-premxied jet flames. Combust. Flame 125:1239-1257

Elsinga, GE, Wieneke, B, Scarano, F, van Oudheusden, BW (2006) Tomographic particle image velocimetry. Exp. Fluids 41:933-947

Elsinga, GE, Wieneke, B, Scarano, F, Schröder, A (2008) Tomographic 3D-PIV and Applications. A. Schroeder, C.E. Willert (Eds.): Particle Image Velocimetry, Topics Appl. Physics 112:103-125
Elsinga, GE, Westerweel, J, Scarano, F, Novara, M (2011) On the velocity of ghost particiles and the bias errors in Tomographic PIV. Exp. Fluids $50: 825-838$

Egolfopoulos, FN (1994) Dynamics and structure of unsteady, strained, laminar premixed flames. Proc. Combust. Inst. 25:1365-1373

Filatyev, SA, Driscoll, JF, Carter, CD, Donbar, JM (2005) Measured properties of turbulent premixed flames for model assessment, including burning velocities, stretch rates and surface densities. Combust. Flame 141:1-21

Foucaut, JM, Stanislas, M (2002) Some considerations on the accuracy and frequency response of some derivative filters applied to particle image velocimetry vector fields. Meas. Sci. Technol. 13:1058-1071

Foucaut, JM, Carlier, J, Stanislas, M (2004) PIV optimization for the study of turbulent flow using spectral analysis. Meas. Sci Technol. 15:10461058

Frank, JH, Kalt, PAM, Bilger, RW (1999) Measurements of conditional velocities in turbulent premixed flames by simultaneous $\mathrm{OH}$ PLIF and PIV. Combust. Flame 116:220-232

Frank, JH, Kaiser, SA, Long, MB (2002) Reactionrate, mixture-fraction, and temperature imaging in turbulent methane/air jet flames. Proc, Combust. Inst. 29:2687-2694

Frank, JH, Kaiser, SA (2010) High-resolution imaging of turbulence structures in jet flames and nonreacting jets with laser Rayleigh scattering. Exp. Fluids 49:823-837 
Gamba, M, Clemens, NT, Ezekoye, OA (2013) Volumetric PIV and 2D OH PLIF imaging in the far-field of a low Reynolds number nonpremixed jet flame. Meas. Sci. Technol. 24:024001 (20pp)

Ganapathisubramani, B, Lakshminarasimhan, K, Clemens, NT (2007) Determination of complete velocity gradient tensor by using cinematographic stereoscopic PIV in a turbulent jet. Exp. Fluids 42:923-939.

Ganapathisubramani, B, Lakshminarasimhan, K, Clemens, NT (2008) Investigation of threedimensional structure of fine scales in a turbulent jet by using cinematographic stereoscopic particle image velocimetry. J. Fluid Mech. 598:141-75

Garcia. D (2010) Robust smoothing of gridded data in one and higher dimensions with missing values. Computational Statistics and Data Analysis 54:1167-1178

Garcia, D (2011) A fast all-in-one method for automated post-processing of PIV data. Exp. Fluids 50:1247-1259

Gomez, A., Rosner, DE (1993) Thermophoretic effects on particles in counterflow laminar diffusion flames. Combust. Sci. and Technol. 89:335-362

Ghaemi, S, Scarano, F (2011) Counter-hairpin vortices in the turbulent wake of a sharp trailing edge. J. Fluid Mech. 689:317-356

Han, D, Mungal, MG (2000) Simultaneous measurement of velocity and $\mathrm{CH}$ layer distribution in turbulent non-premixed flames. Proc. Combust. Inst. 28:261-267

Han, D, Mungal, MG (2003) Simultaneous measurements of velocity and $\mathrm{CH}$ distributions.
Part 1: jet flames in co-flow. Combust. Flame $132: 565-590$

Hearst, RJ, Buxton, ORH, Ganapathisubramani, B, Lavoie, P (2012) Experimental estimation of fluctuating velocity and scalar gradients in turbulence. Exp. Fluids 53:925-942

Kaiser, SA, Frank, JH, Long, MB (2005) Use of Rayleigh imaging and ray tracing to correct for beam-steering effects in turbulent flames. Appl. Opt. 44:6557-6564

S. A. Kaiser, J. H. Frank, Spatial scales of extinction and dissipation in the near field of non-premixed turbulent jet flames. Proc. Combust. Inst. 32:16391646 (2009).

Keane, RD, Adrian, RJ (1990) Optimization of particle image velocimetry. Part I: Double pulsed systems. Meas. Sci. Technol. 1:1202-1215

Kee, R.J., Rupley, F.M., Miller, J.A., Coltrin, M.E., Grcar, J.F., Meeks, E., Moffat, H.K., Lutz, A.E., Dixon-Lewis, G., Smooke, M.D., Warnatz, J., Evans, G.H., Larson, R.S., Mitchell, R.E., Petzold, L.R., Reynolds, W.C., Caracotsios, M., Stewart, W.E., Glarborg, P., Wan, C., McLellan, C.L., Adigun, O., Houf, W.G., Chou, C.P., Miller, S.F., Ho, P., Young, P.D., Young, D.J., Hodgson, D.W., Petrova, M.V., Puduppakkam, K.V. (2007). CHEMKIN Collection, Release 4.1.1, Reaction Design, Inc., San Diego, CA

Kim, M, Yoon, Y (2007) Flame residence time and strain rate in turublent hydrogen non-premixed jet flames with coaxial air. Proc. Combust. Inst. 31:1609-1616

Kothnur, PS, Tsurikov, MS, Clemens, NT, Donbar, JM, Carter, CD (2002) Planar imaging of $\mathrm{CH}, \mathrm{OH}$ 
and velocity in turbulent non-premixed jet flames. Proc. Combust. Inst. 29:1921-1927

Lavoie, P, Avallone, G, De Gregorio, F, Romano, GP, Antonia, RA (2007) Spatial resolution of PIV for the measurement of turbulence. Exp. Fluids 43:3951

Mullin, JA, Dham, WJA (2006) Dual-plane stereo particle image velocimetry measurements of velocity gradient tensor fields in turbulent shear flow. I. Accuracy assessments. Phys. Fluids 18:035101

Prasad, AK, Adrian, RJ (1993) Stereoscopic particle image velocimetry applied to liquid flows. Exp. Fluids 15:49-60

Rehm, JE, Clemens, NT (1998) The relationship between vorticity/strain and reaction zone structure in turbulent non-premixed jet flames. Proc. Combust. Inst. 27:1113-1120

Rhem, JE, Clemens, NT (1999) The association of scalar dissipation rate layers and $\mathrm{OH}$ zones with strain, vorticity, and 2-D dilation fields in turbulent nonpremixed jets and jet flames. AIAA99-0676, $37^{\text {th }}$ Aerospace Sciences Meeting

Scarano, F (2003) Theory of non-isotropic spatial resolution in PIV. Exp. Fluids 35:268-277

Scarano, F, Poelma, C (2009) Three-dimensional vorticity patterns of cylinder wakes. Exp. Fluids 47:69-83

Scarano, F (2013) Tomographic PIV: principles and practice. Meas. Sci. Technol. 24:012001 (28pp)

Schefer, RW, Goix, PJ (1998) Mechanism of flame stabilization in turbulent, lifted-jet flames. Combust. Flame 112:559-574
Sick, V (2013) High speed imaging in fundamental and applied combustion research. Proc. Combust. Inst. 34:3509-3530

Soira, J, Willert, C (2012) On measuring the joint probability desnity function of three-dimensional velocity components in turbulent flows. Meas. Sci. Technol. 23:065301

Steinberg, AM, Driscoll, JF, Ceccio, SL (2009) Threedimensional temporally resolved measurements of turbulence-flame interactions using orthogonalplane cinema-stereoscopic PIV. Exp. Fluids, 47:527-47

Steinberg, AM, Boxx, I, Arndt, CM, Frank, JH, Meier, W (2011) Experimental study of flame-hole reignition mechanism in a turbulent non-premixed jet flame using sustained multi-kHz PIV and crossed-plane OH PLIF. Proc. Combust. Inst. 33:1663-1672

TNF Workshop, http://www.ca.sandia.gov/TNF, Barlow, RS, Eds., Sandia National Laboratories.

Tsurikov, MS, Clemens, NT (2002) The structure of dissipative scales in axisymmetric turbulent gasphase jets. AIAA 2002-0164

Wang, G, Karpetis, AN, Barlow, RS (2007) Dissipation length scales in turbulent nonpremixed jet flames. Combust. Flames 148:62-75

Weinkauff, J., Michaelis, D., Dreizler, A., Böhm, B (2013) Tomographic PIV measurements in a turbulent lifted jet flame. Exp. Fluids 54:1624

Westerweel, J, Scarano, F (2005) Universal outlier detection for PIV data. Exp. in Fluids 39:10961100 
Westerweel, J (2008) On velocity gradients in PIV interrogation. Exp. Fluids 44:831-842.

Wieneke, B (2008) Volume self-calibration for 3D particle image velocimetry. Exp. fluids 45:549556

Worth, NA, Nickels, TB, Swaminathan, N (2010) A tomographic PIV resolution study based on homogeneous isotropic turbulence DNS data. Exp Fluids 49:637-656
Zhang, J, Tao, B, Katz, J (1997) Turbulent flow measurement in a square duct with hybrid holographic PIV. Exp. Fluids 23:373-381

Zhao, Z, Chaos, M, Kazakov, A, Dryer, FL (2008) Thermal decomposition reaction and a comprehensive kinetic model of dimethyl ether. Int. J. Chem. Kin. 40:1-18 


\section{List of Supplemental Materials}

Online Resource 1 Simultaneous $10 \mathrm{kHz}$ TPIV and OH PLIF measurements obtained in the stabilization region of a lifted jet flame. Blue isosurfaces are for $\omega^{2}=15 \times 10^{6} \mathrm{~s}^{-2}$. Velocity vectors are represented in the same plane as the OH PLIF images (1 out of 16 in-plane vectors displayed).

Online Resource 2 Simultaneous $10 \mathrm{kHz}$ TPIV and OH-PLIF measurements in the turbulent partially premixed

DME/air jet flame of Fig. $2 \mathrm{~b}$ at $y / D=20$. Blue isosurfaces are for $\Gamma_{o}=-15 \times 10^{3} \mathrm{~s}^{-1}$. Velocity vectors are represented in the same plane as the OH PLIF images (1 out of 16 in-plane vectors displayed). 


\section{List of Figures}

Fig. 1 Experimental configuration for simultaneous high-repetition rate tomographic PIV and OH PLIF

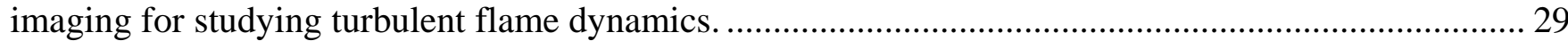
Fig. 2 Photographs of the piloted jet burner and flame luminosity from the DME/air jet flames: (a) weakly-turbulent, partially-premixed lifted jet flame (Case 1) and (b) turbulent partially-premixed jet flame (Case 2). Solid rectangles indicate the locations of simultaneous $10 \mathrm{kHz}$ TPIV and OH PLIF measurements, and the dashed rectangle is the location of the $16 \mathrm{kHz}$ TPIV measurements. $16 \mathrm{kHz}$ TPIV measurements were also performed in a non-reactive turbulent jet at the same location.

Fig. 3 (a) Instantaneous particle distribution in a reconstructed probe volume. Particle images are displayed only on the six surfaces of the probe volume. Particles are identified using thresholding transparency of the Mie-scattering signal. (b) Corresponding 3D velocity vector field. Velocity vectors are scaled and color-coded according to their magnitude. Only 1 out of 64 vectors are displayed for clarity. The main jet flow is on the right-hand side, and the lifted flame is stabilized towards the upper left of the probe volume in the region of lower particle density.

Fig. 4 Time sequences from a turbulent air jet (Case 2NR): (a) Apparent divergence squared indicates locations of errors in measurements of $(\nabla \cdot v)^{2}$. Orange isosurfaces indicate $(\nabla \cdot v)^{2}=500 \times 10^{6} \mathrm{~s}^{-2} \cdot(\mathrm{b})$ Enstrophy and divergence squared shows persistence of coherent structures of high vorticity and the lack of persistence in the apparent divergence. Blue isosurfaces correspond to $\omega^{2}=500 \times 10^{6} \mathrm{~s}^{-2} \ldots \ldots \ldots \ldots \ldots 31$

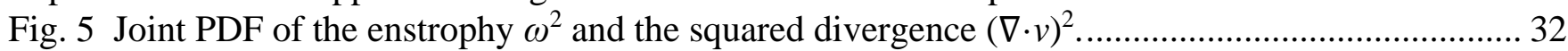
Fig. 6 Effect of TPIV interrogation volume size and vector spacing, $\Delta X$, on the probability density function of apparent divergence in the turbulent air jet at a downstream position of $y / D=10$............ 32 Fig. 7 Effect of smoothing strength parameter, $s$, on enstrophy (blue) and squared divergence (orange) isosurfaces for (a) $\omega^{2}=(\nabla \cdot v)^{2}=1,000 \times 10^{6} \mathrm{~s}^{-2}$ and (b) $\omega^{2}=(\nabla \cdot v)^{2}=500 \times 10^{6} \mathrm{~s}^{-2}$ in the turbulent air jet at

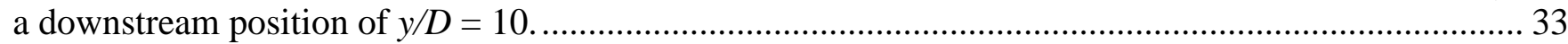

Fig. 8 Effects of smoothing strength on fluctuations of (a) velocity (b) enstrophy and squared divergence in the turbulent air jet. Fluctuations are defined as the temporal standard deviation of a quantity, averaged over the probe volume.

Fig. 9 Time sequence of simultaneous TPIV velocity field and OH PLIF measurements in the stabilization region of a lifted jet flame. The time interval between the displayed snapshots is $0.5 \mathrm{~ms}$. Blue surfaces are enstrophy isosurfaces for $\omega^{2}=15 \times 10^{6} \mathrm{~s}^{-2}$. Velocity vectors are plotted in the same plane as the OH PLIF images (1 out of 16 in-plane vectors displayed).

Fig. 10 Tracking of the vortex tube identified in the red box in Fig. 9 (time: $0.0 \mathrm{~ms}$ ). Blue surfaces are enstrophy isosurfaces for $\omega^{2}=15 \times 10^{6} \mathrm{~s}^{-2}$

Fig. 11 Evolution of the enstrophy within the core of the vortex tube in Fig. 9 and the distance of the vortex with respect to the flame front. Images show the enstrophy in the vortex in the midplane at $z=0.0 \mathrm{~mm}$.

Fig. 12 Comparison of vortical structures in (a) non -reactive (Case 2NR) and (b) burning (Case 2) turbulent jet at $y / D=10$. Blue surfaces are enstrophy isosurfaces for $\omega^{2}=500 \times 10^{6} \mathrm{~s}^{-2}$. Velocity vectors are displayed in the midplane at $z=0.0 \mathrm{~mm}$ ( 1 out of 16 in-plane vectors displayed).

Fig. 13 Time sequence of simultaneous TPIV and OH-PLIF measurements at $10 \mathrm{kHz}$ in the turbulent partially premixed DME/air jet flame at $y / D=20$. Blue surfaces are isosurfaces of the compressive strain rate for $\Gamma_{o}=-15 \times 10^{3} \mathrm{~s}^{-1}$. Velocity vectors are shown in the same plane as the OH PLIF images (1

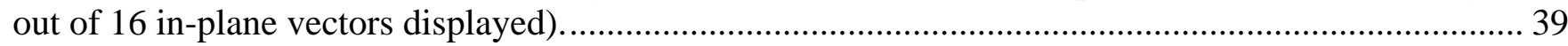
Fig. 14 Single shot measurement from the time sequence in Fig. 13 with the principal axes of the compressive strain rate plotted for $\Gamma_{o} \leq-15 \times 10^{3} \mathrm{~s}^{-1}$.... 40

Fig. 15 Comparison of compressive strain rate statistics in non-reactive (Case $2 N R$ - blue curves) and reactive (Case 2 - red curves) turbulent jets at $y / D=10$. (a) Distribution of the deviatoric (solid curves), $\Gamma_{o}$, and non-deviatoric (dashed curves), $\Gamma$, compressive strain rate. (b) Distribution of the deviatoric 
compressive strain rate orientation for $\Gamma_{o} \leq-15 \times 10^{3} \mathrm{~s}^{-1}$ in the $x y$ (solid curves) and $z y$ (dashed curves) planes.

Fig. 16 Joint PDFs of (a) 2D and 3D non-deviatoric compressive strain rates and (b) 2D compressive strain rate and orientation of the compressive strain principal axis in the $z y$ plane. Data are from the turbulent jet flame (Case 2) at $\mathrm{y} / \mathrm{D}=10$. 
Fig. 1 Experimental configuration for simultaneous high-repetition rate tomographic PIV and OH PLIF imaging for studying turbulent flame dynamics.

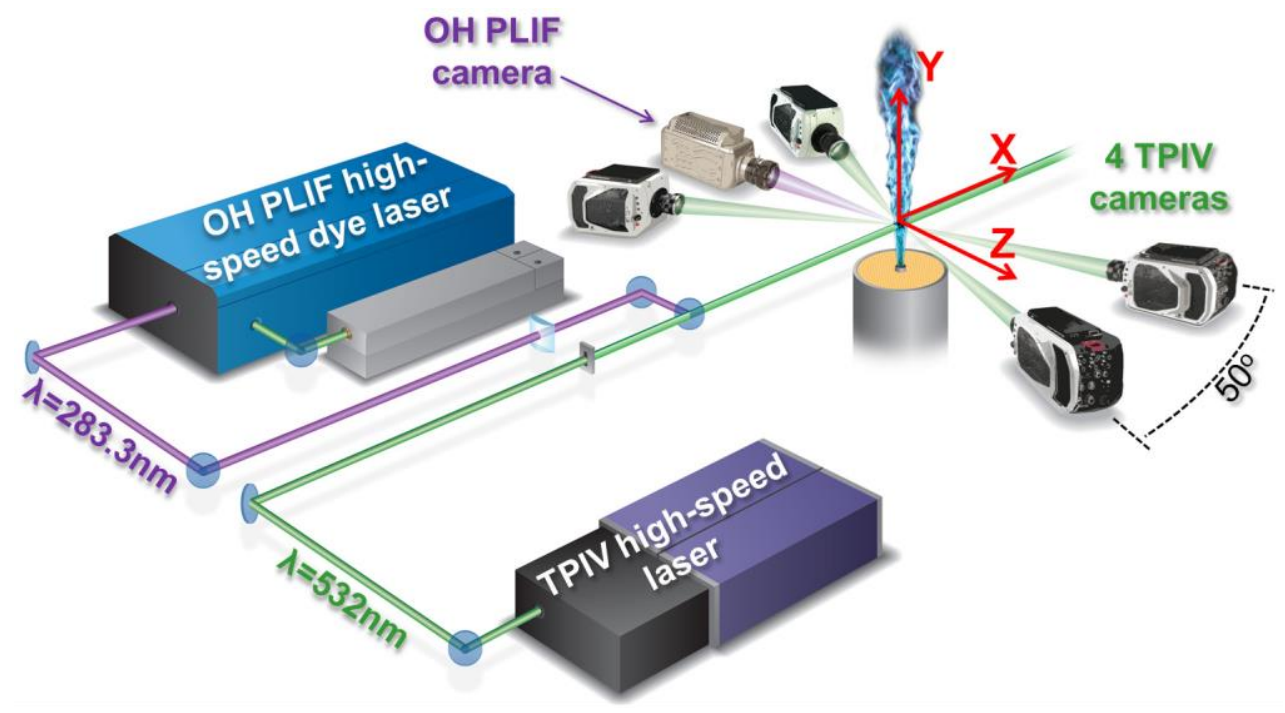

Fig. 2 Photographs of the piloted jet burner and flame luminosity from the DME/air jet flames: (a) weaklyturbulent, partially-premixed lifted jet flame (Case 1) and (b) turbulent partially-premixed jet flame (Case 2). Solid rectangles indicate the locations of simultaneous $10 \mathrm{kHz}$ TPIV and OH PLIF measurements, and the dashed rectangle is the location of the $16 \mathrm{kHz}$ TPIV measurements. $16 \mathrm{kHz}$ TPIV measurements were also performed in a non-reactive turbulent jet at the same location.

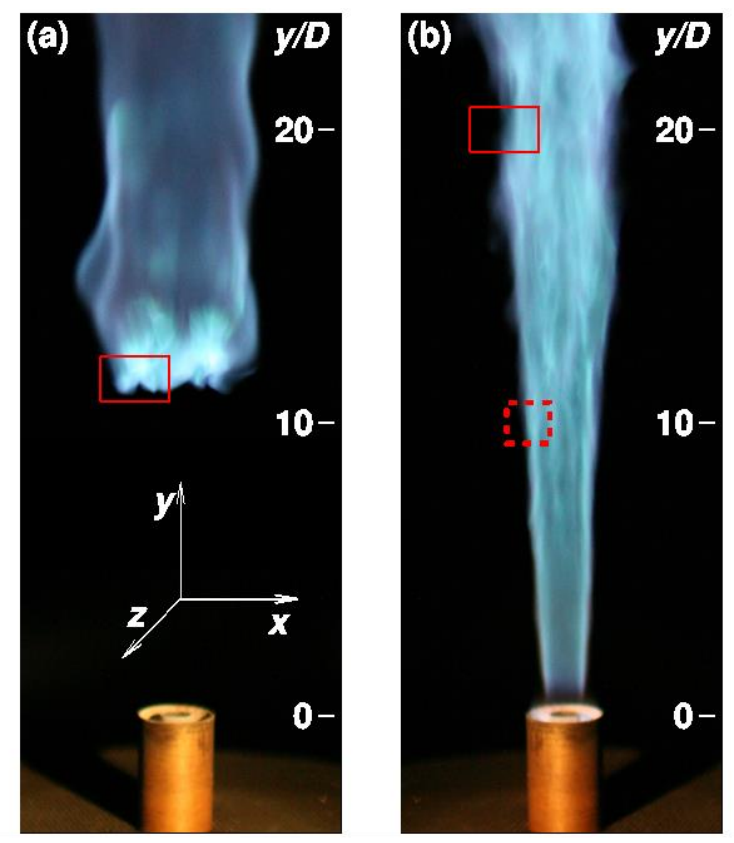


Fig. 3 (a) Instantaneous particle distribution in a reconstructed probe volume. Particle images are displayed only on the six surfaces of the probe volume. Particles are identified using thresholding transparency of the Mie-scattering signal. (b) Corresponding 3D velocity vector field. Velocity vectors are scaled and color-coded according to their magnitude. Only 1 out of 64 vectors are displayed for clarity. The main jet flow is on the right-hand side, and the lifted flame is stabilized towards the upper left of the probe volume in the region of lower particle density.

(a)
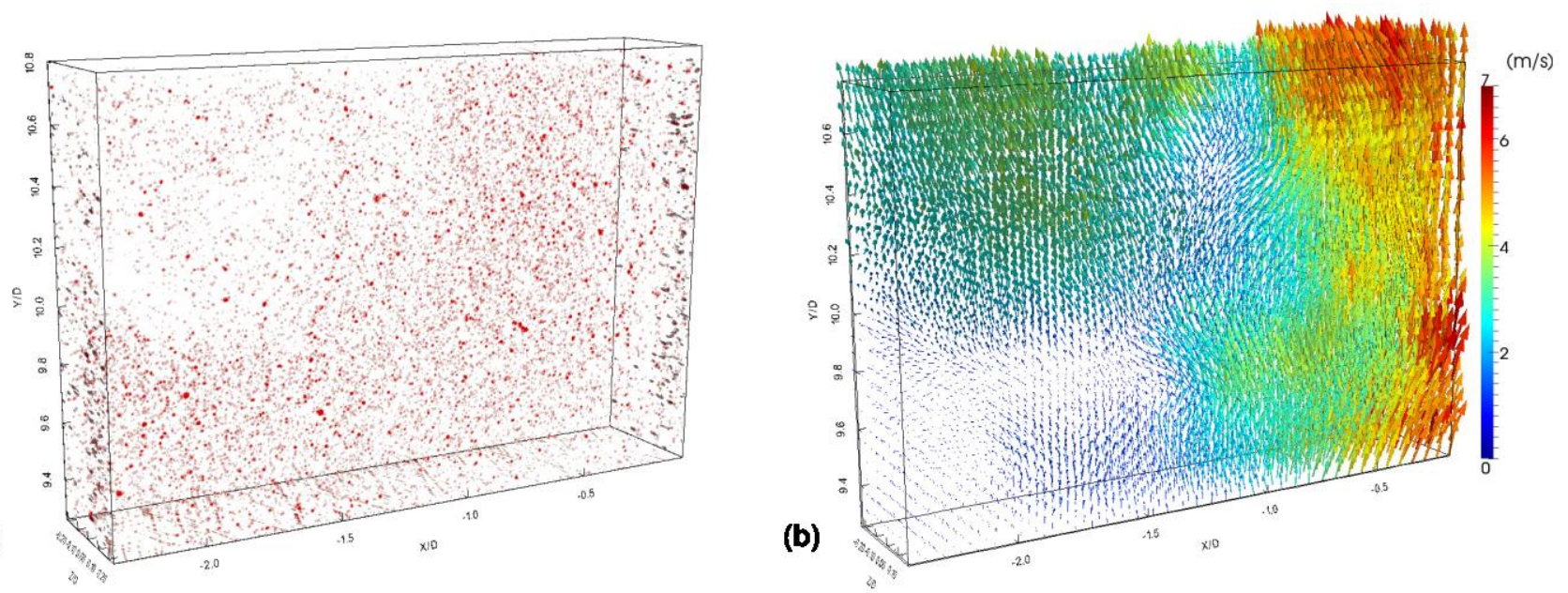
Fig. 4 Time sequences from a turbulent air jet (Case 2NR): (a) Apparent divergence squared indicates locations of errors in measurements of $(\nabla \cdot v)^{2}$. Orange isosurfaces indicate $(\nabla \cdot v)^{2}=500 \times 10^{6} \mathrm{~s}^{-2}$.(b) Enstrophy and divergence squared shows persistence of coherent structures of high vorticity and the lack of persistence in the apparent divergence. Blue isosurfaces correspond to $\omega^{2}=500 \times 10^{6} \mathrm{~s}^{-2}$

(a)
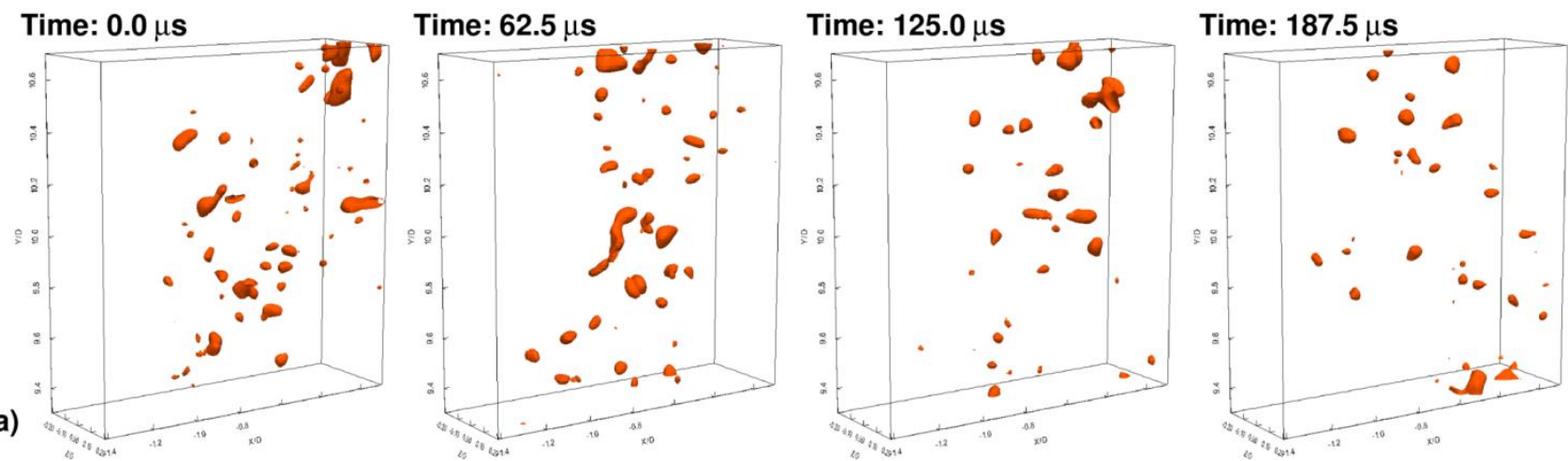

Time: $0.0 \mu \mathrm{s}$

(b)

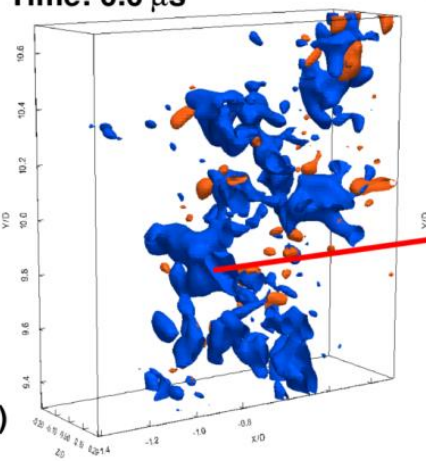

Time: $62.5 \mu \mathrm{s}$

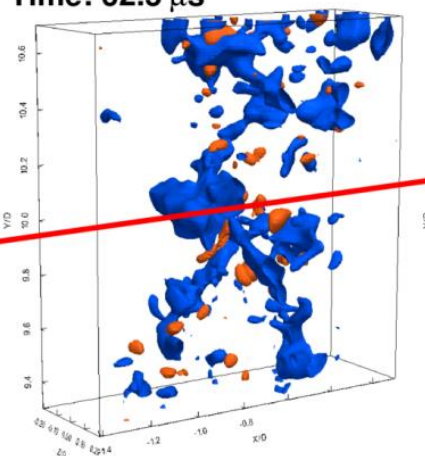

Time: $125.0 \mu \mathrm{s}$

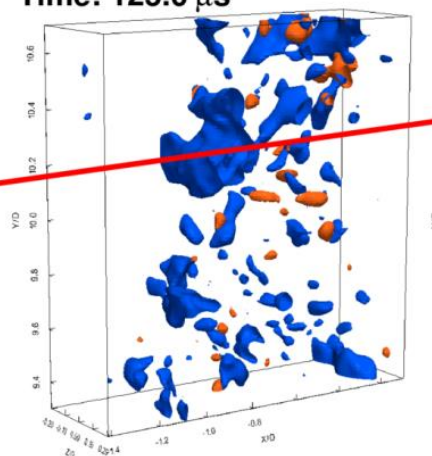

Time: $187.5 \mu \mathrm{s}$

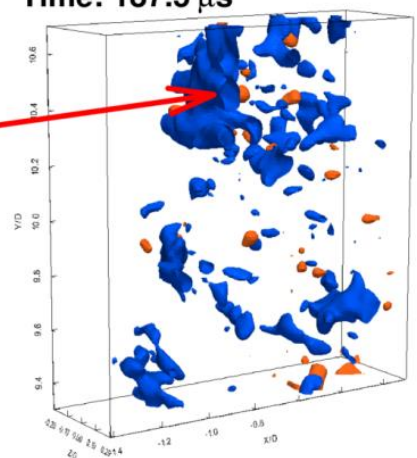


Fig. 5 Joint PDF of the enstrophy $\omega^{2}$ and the squared divergence $(\nabla \cdot v)^{2}$.

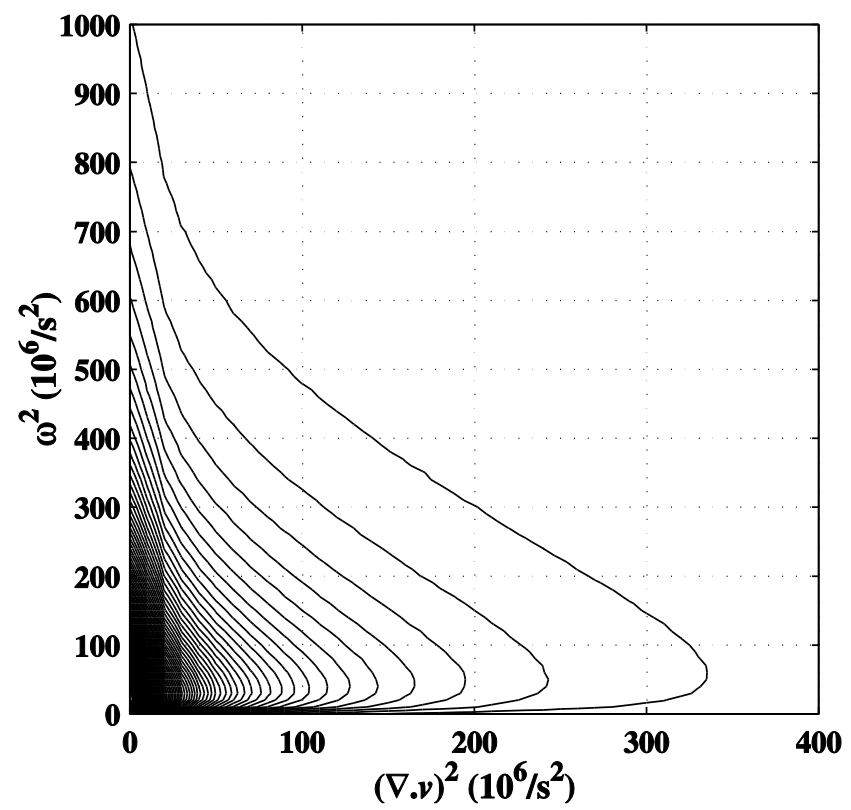

Fig. 6 Effect of TPIV interrogation volume size and vector spacing, $\Delta X$, on the probability density function of apparent divergence in the turbulent air jet at a downstream position of $y / D=10$.
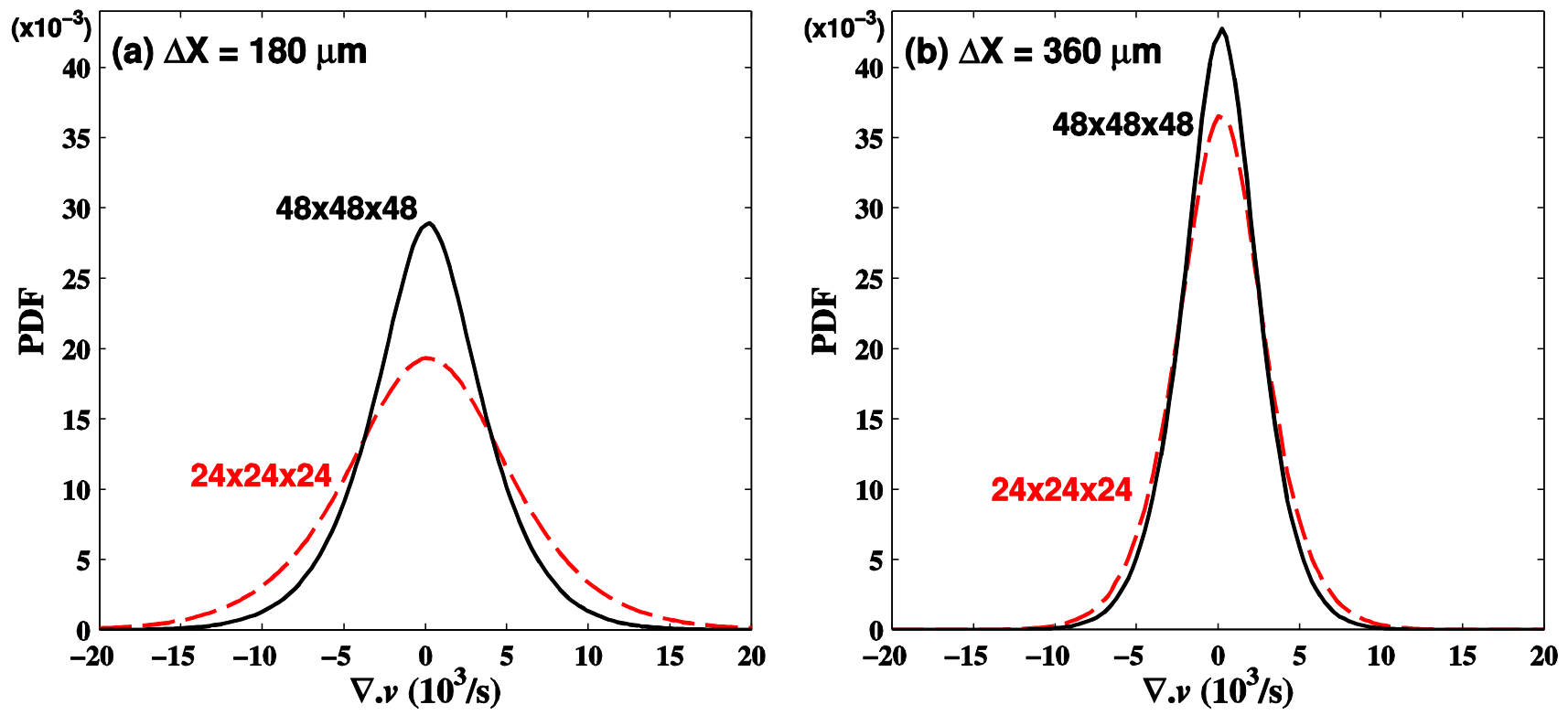
Fig. 7 Effect of smoothing strength parameter, $s$, on enstrophy (blue) and squared divergence (orange) isosurfaces for (a) $\omega^{2}=(\nabla \cdot v)^{2}=1,000 \times 10^{6} \mathrm{~s}^{-2}$ and $(\mathrm{b}) \omega^{2}=(\nabla \cdot v)^{2}=500 \times 10^{6} \mathrm{~s}^{-2}$ in the turbulent air jet at a downstream position of $y / D=10$.

(a)
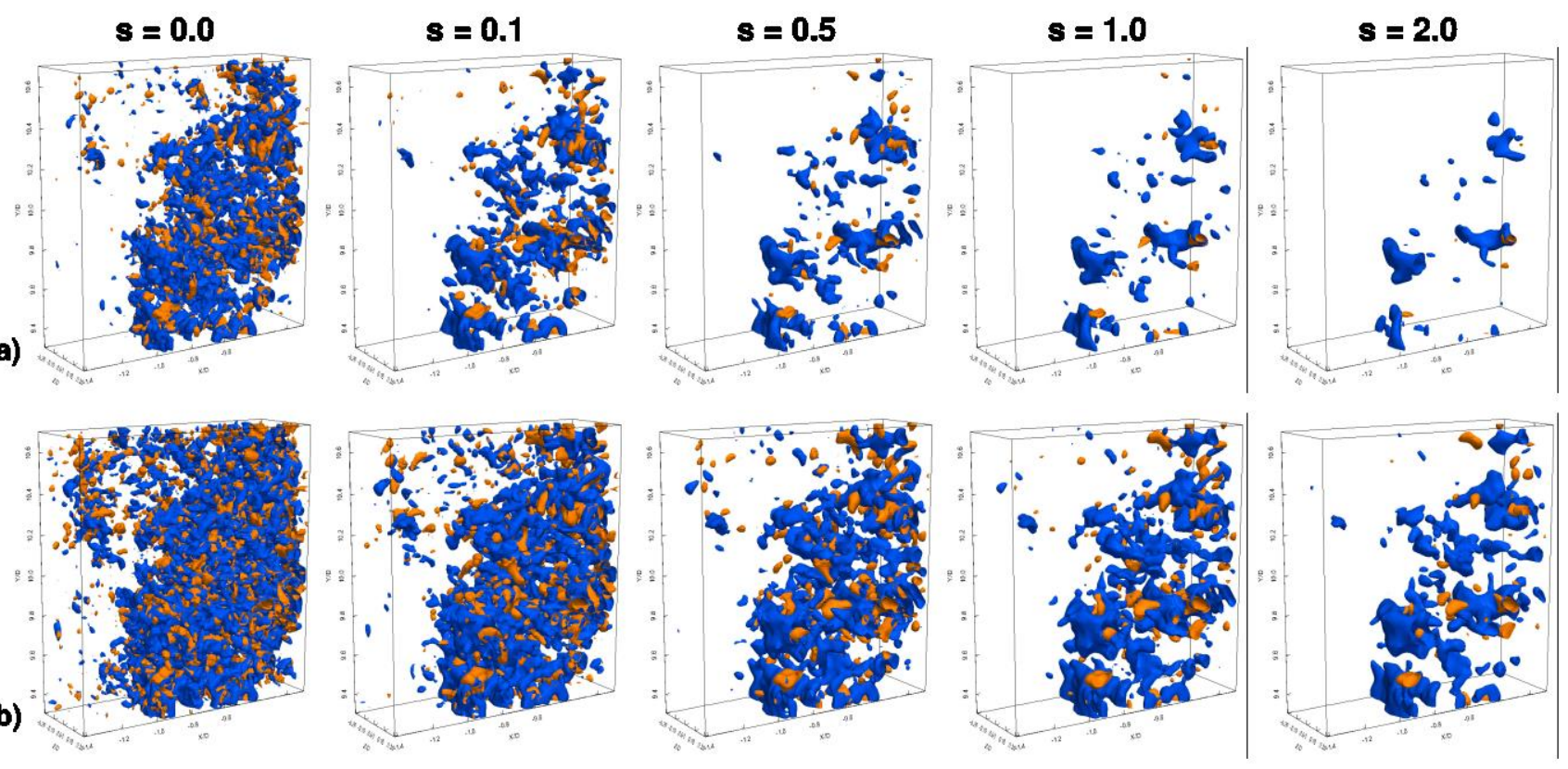
Fig. 8 Effects of smoothing strength on fluctuations of (a) velocity (b) enstrophy and squared divergence in the turbulent air jet. Fluctuations are defined as the temporal standard deviation of a quantity, averaged over the probe volume.
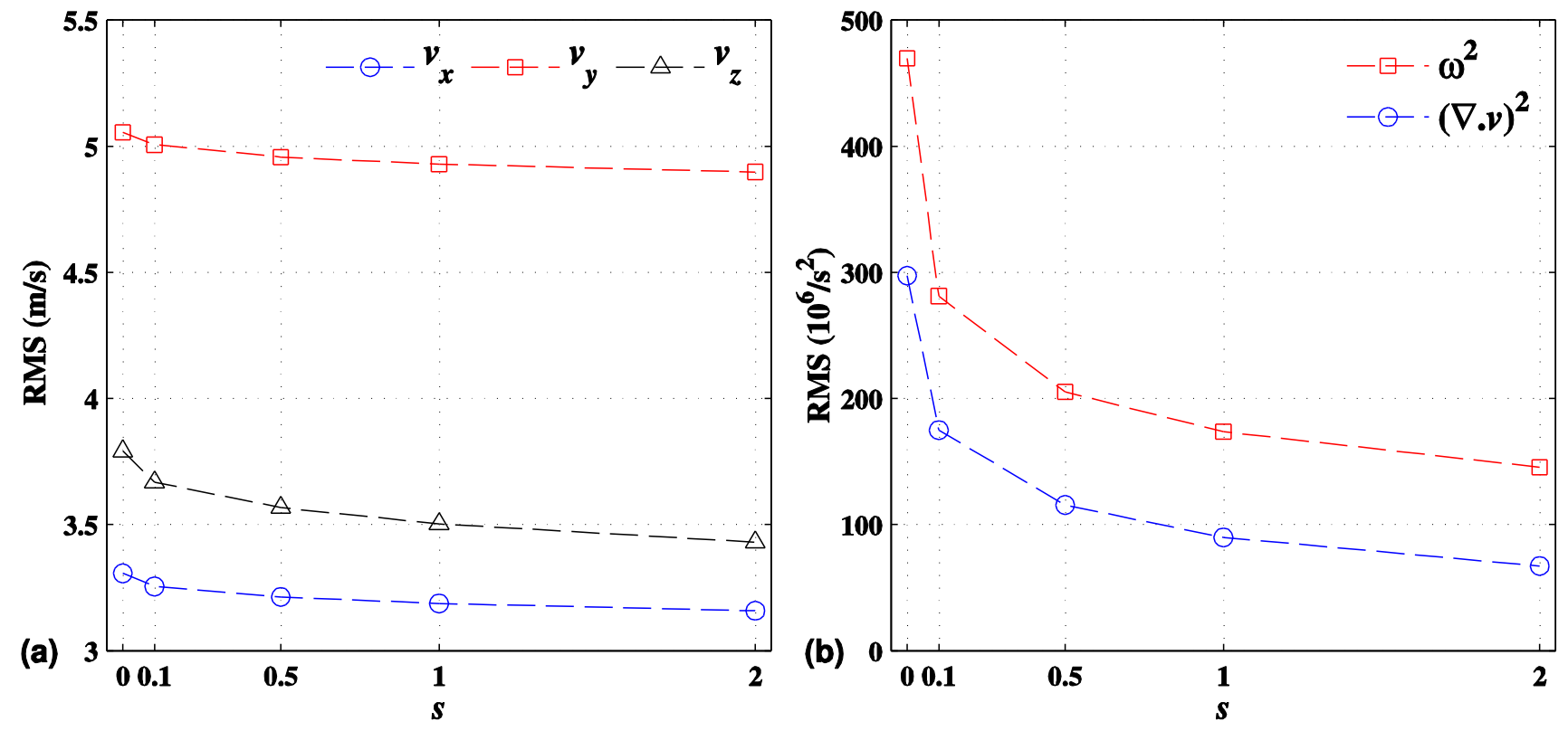
Fig. 9 Time sequence of simultaneous TPIV velocity field and OH PLIF measurements in the stabilization region of a lifted jet flame. The time interval between the displayed snapshots is $0.5 \mathrm{~ms}$. Blue surfaces are enstrophy isosurfaces for $\omega^{2}=15 \times 10^{6} \mathrm{~s}^{-2}$. Velocity vectors are plotted in the same plane as the OH PLIF images (1 out of 16 in-plane vectors displayed).

Fig. 10 Tracking of the vortex tube identified in the red box in Fig. 9 (time: $0.0 \mathrm{~ms}$ ). Blue surfaces are enstrophy isosurfaces for $\omega^{2}=15 \times 10^{6} \mathrm{~s}^{-2}$.

Fig. 11 Evolution of the enstrophy within the core of the vortex tube in Fig. 9 and the distance of the vortex with respect to the flame front. Images show the enstrophy in the vortex in the midplane at $z=0.0 \mathrm{~mm}$.

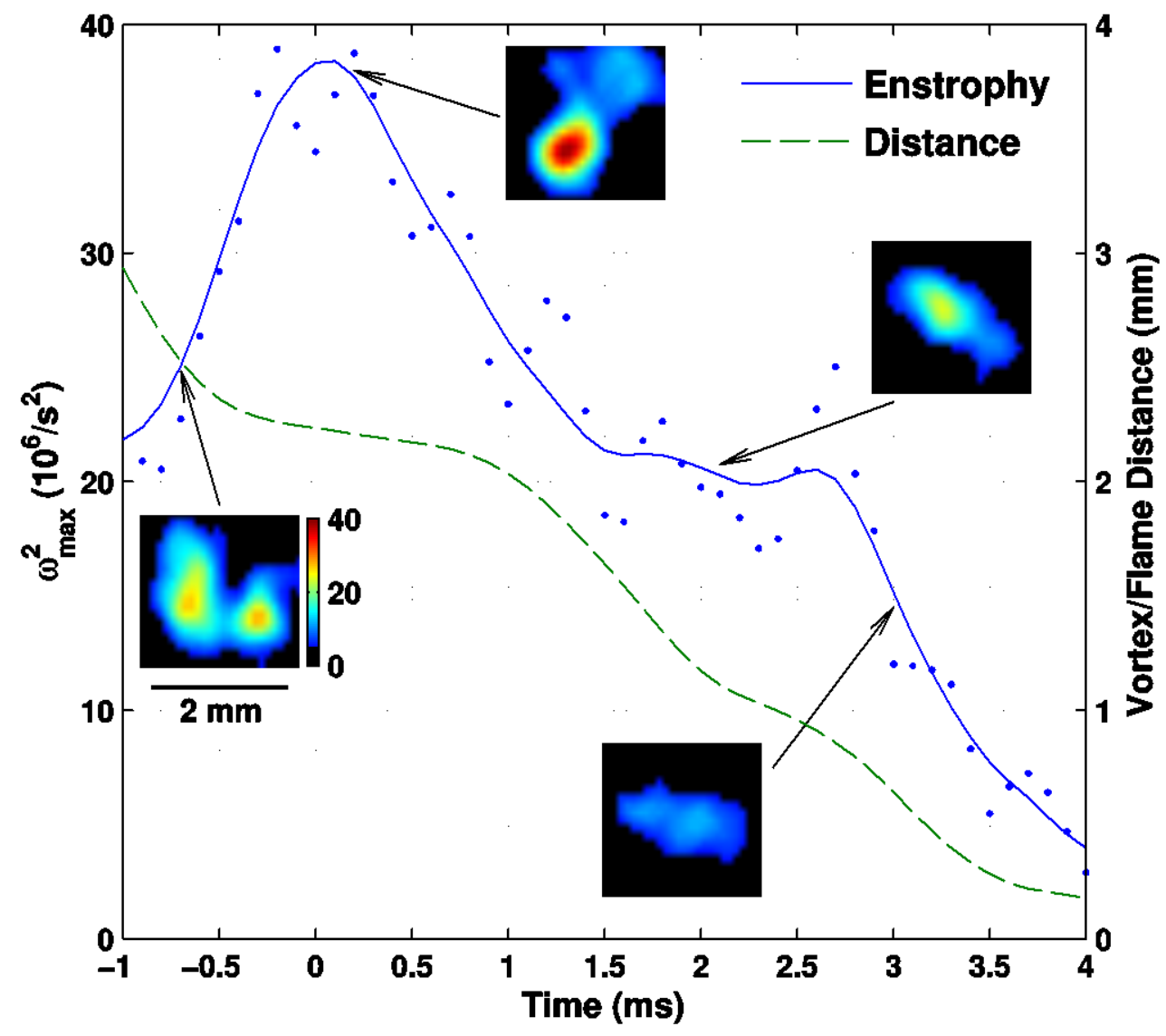


Fig. 12 Comparison of vortical structures in (a) non -reactive (Case 2NR) and (b) burning (Case 2) turbulent jet at $y / D=10$. Blue surfaces are enstrophy isosurfaces for $\omega^{2}=500 \times 10^{6} \mathrm{~s}^{-2}$. Velocity vectors are displayed in the midplane at $z=0.0 \mathrm{~mm}$ (1 out of 16 in-plane vectors displayed).

(a)
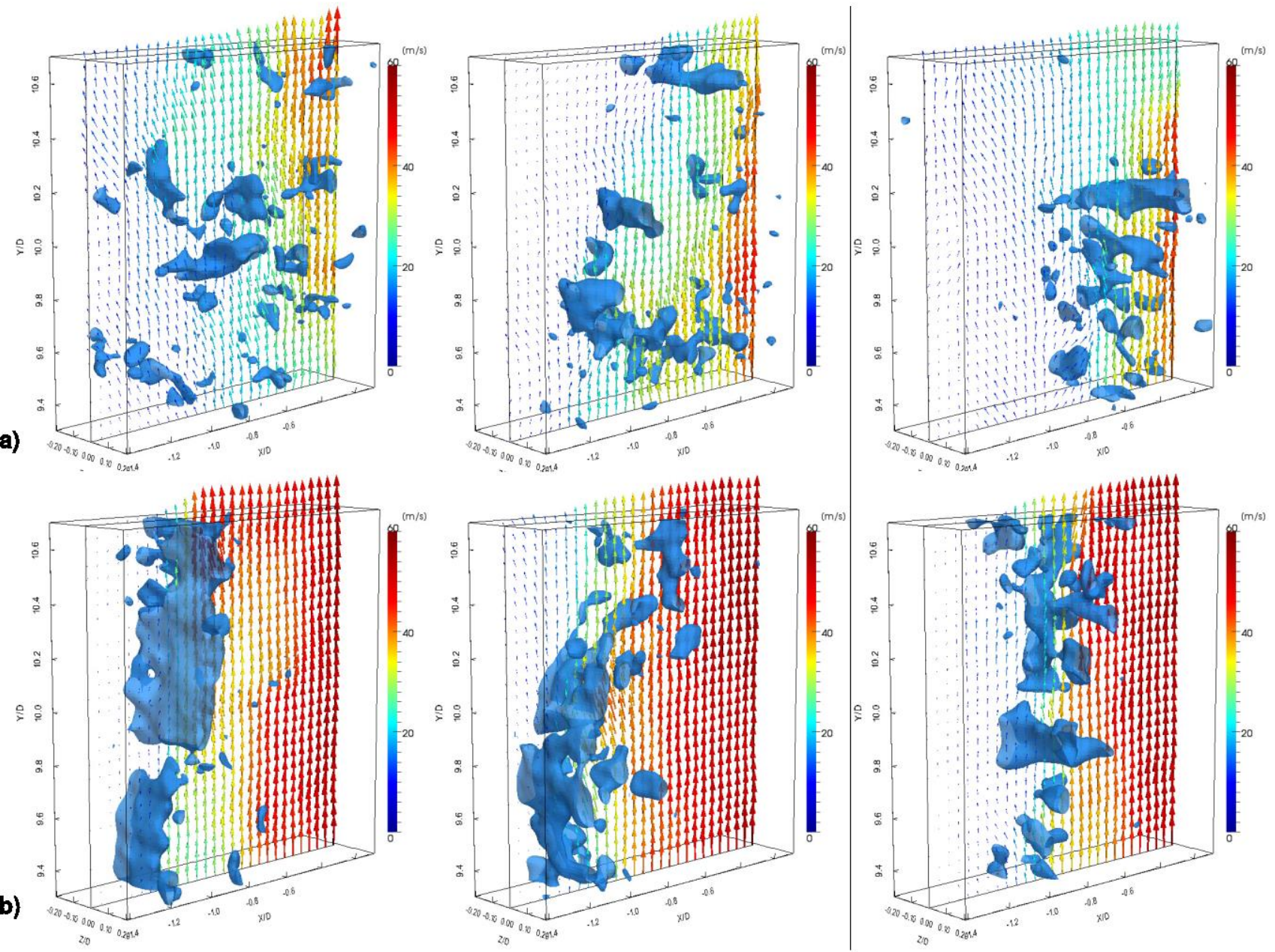
Fig. 13 Time sequence of simultaneous TPIV and OH-PLIF measurements at $10 \mathrm{kHz}$ in the turbulent partially premixed DME/air jet flame at $y / D=20$. Blue surfaces are isosurfaces of the compressive strain rate for $\Gamma_{o}=-15 \times 10^{3} \mathrm{~s}^{-1}$. Velocity vectors are shown in the same plane as the OH PLIF images (1 out of 16 in-plane vectors displayed).

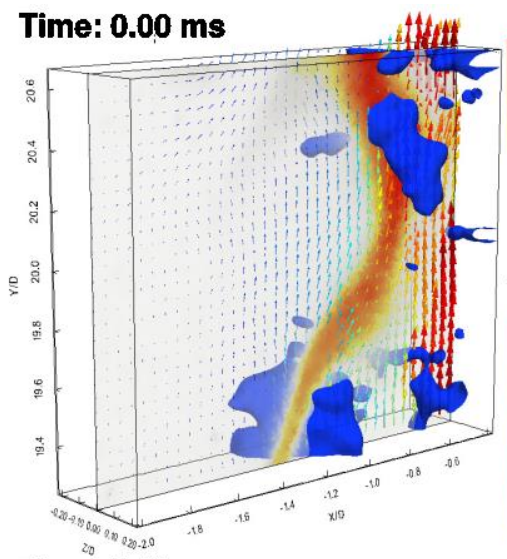

Time: $\mathbf{0 . 3 0} \mathrm{ms}$
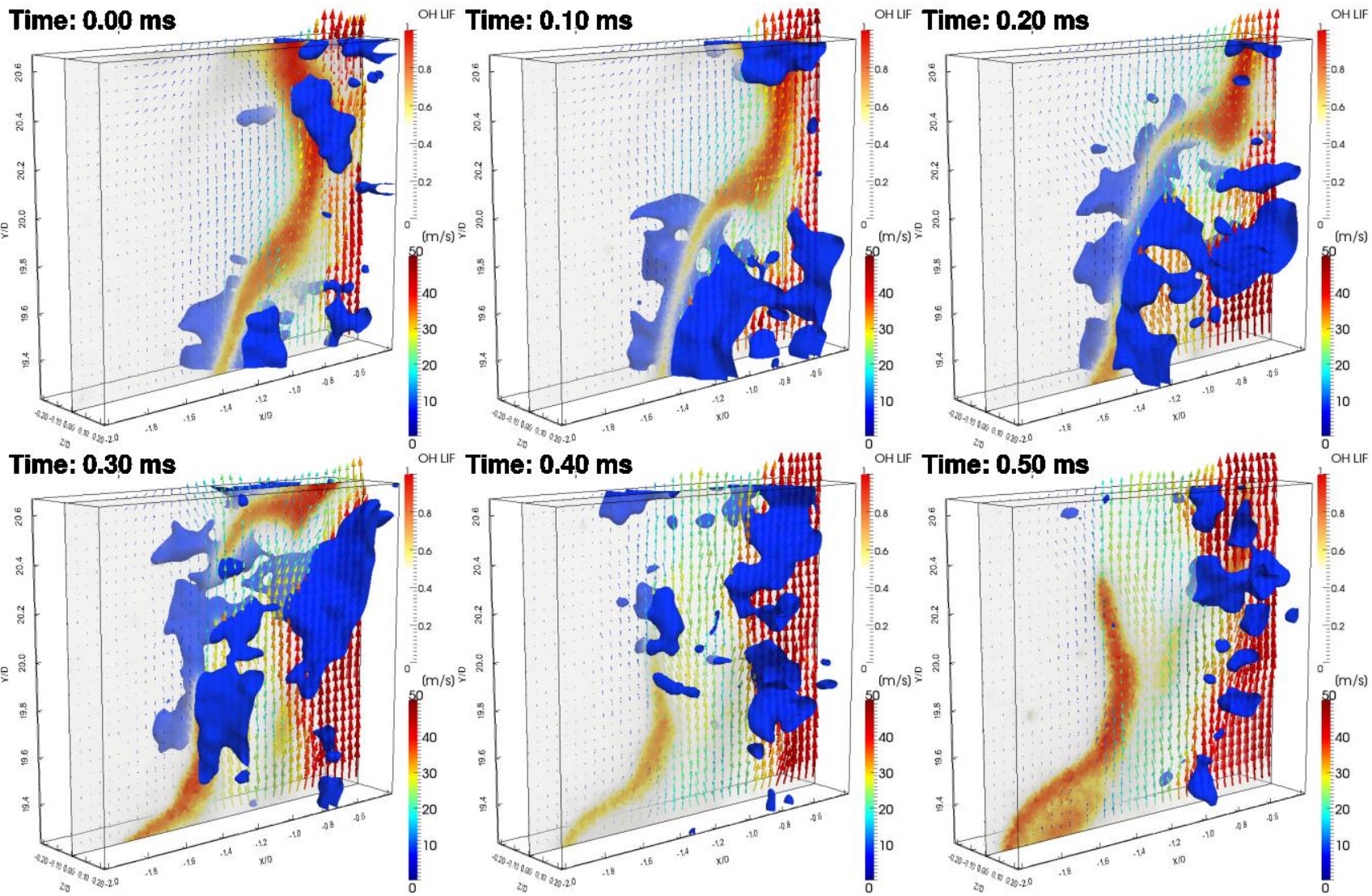
Fig. 14 Single shot measurement from the time sequence in Fig. 13 with the principal axes of the compressive strain rate plotted for $\Gamma_{o} \leq-15 \times 10^{3} \mathrm{~s}^{-1}$.

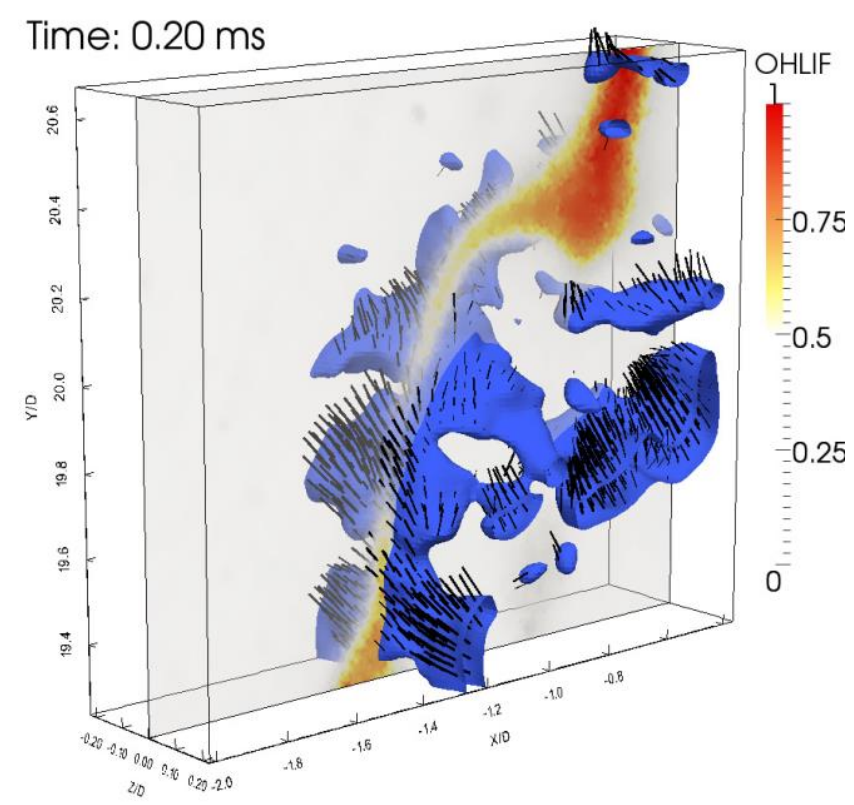


Fig. 15 Comparison of compressive strain rate statistics in non-reactive (Case $2 N R$ - blue curves) and reactive (Case 2 - red curves) turbulent jets at $y / D=10$. (a) Distribution of the deviatoric (solid curves), $\Gamma_{o}$, and non-deviatoric (dashed curves), $\Gamma$, compressive strain rate. (b) Distribution of the deviatoric compressive strain rate orientation for $\Gamma_{o} \leq-15 \times 10^{3} \mathrm{~s}^{-1}$ in the $x y$ (solid curves) and $z y$ (dashed curves) planes.
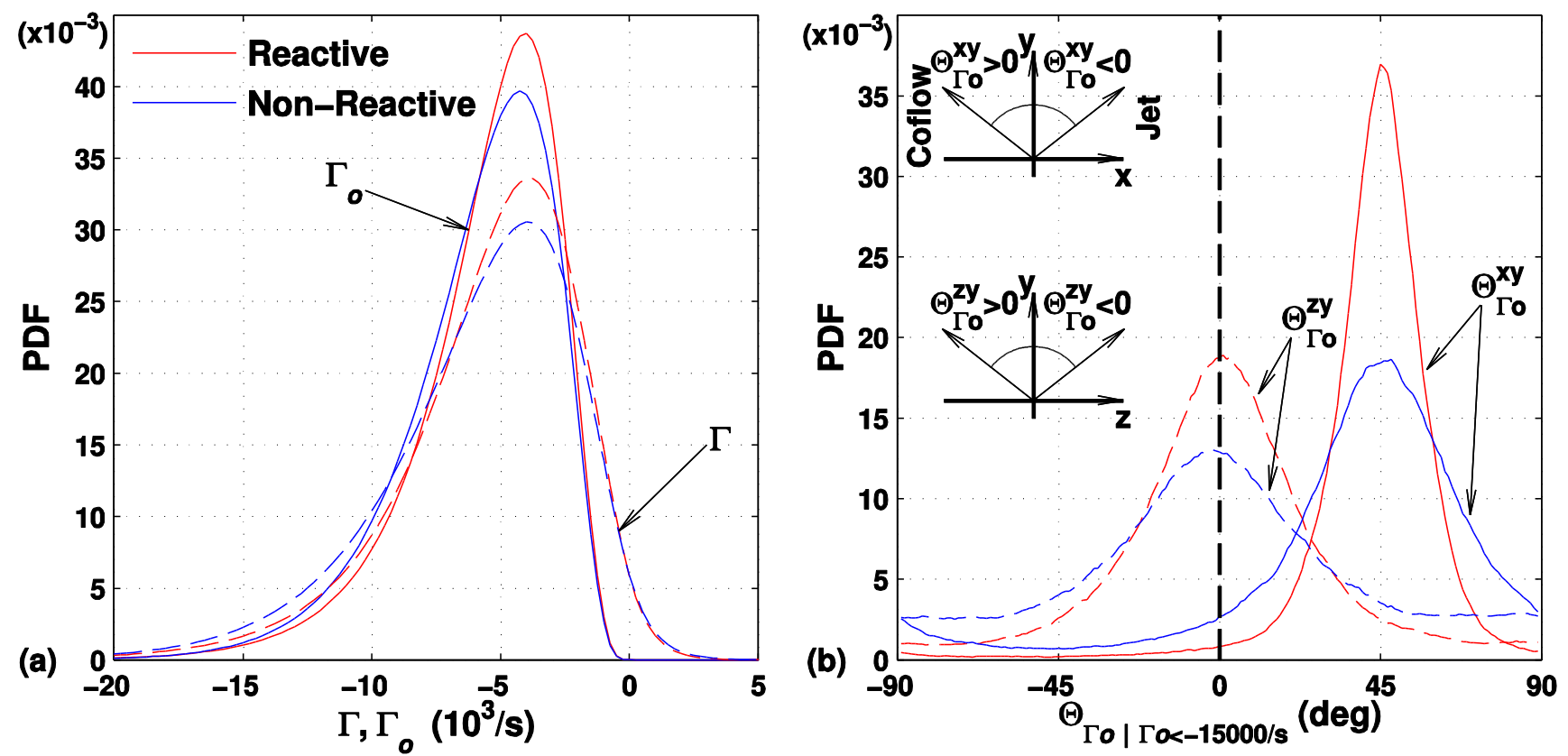

Fig. 16 Joint PDFs of (a) 2D and 3D non-deviatoric compressive strain rates and (b) 2D compressive strain rate and orientation of the compressive strain principal axis in the $z y$ plane. Data are from the turbulent jet flame (Case 2) at $\mathrm{y} / \mathrm{D}=10$.
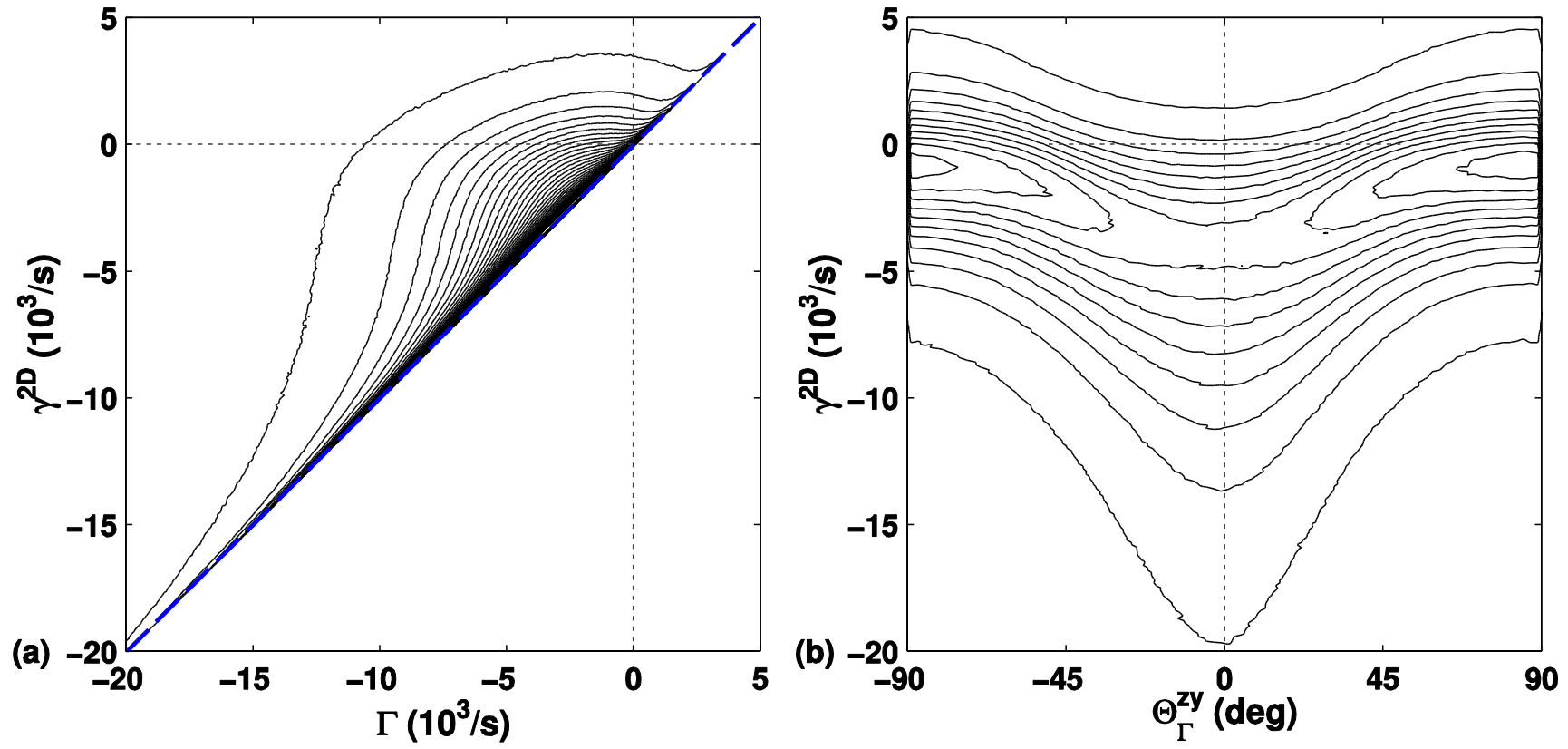